\begin{tabular}{|c|c|}
\hline Title & Ground States of a General Class of Quantum Field Hamiltonians \\
\hline Author(s) & A rai, A sao; Hirokawa, Masao \\
\hline Citation & $\begin{array}{l}\text { Reviews in Mathematical Physics, 12(8), } 1085-1135 \\
\text { https:/doi.org/10.1142/S0129055X 00000393 }\end{array}$ \\
\hline Issue Date & $2000-08$ \\
\hline Doc URL & http:/hdl.handle.net/2115/38267 \\
\hline Rights & 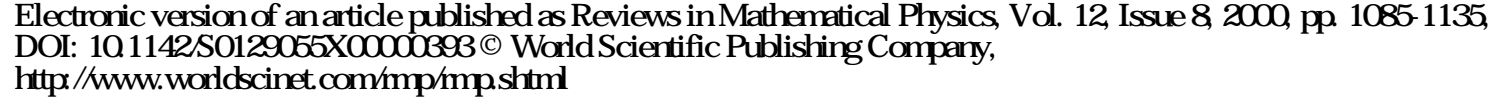 \\
\hline Type & article (author version) \\
\hline File Information & ArHi2.pdf \\
\hline
\end{tabular}

Instructions for use 


\title{
Ground States of a General Class of Quantum Field Hamiltonians
}

\author{
Asao Arai $^{1}$ and Masao Hirokawa ${ }^{2}$ \\ ${ }^{1}$ Department of Mathematics, Hokkaido University, Sapporo 060-0810, Japan, \\ e-mail: arai@math.sci.hokudai.ac.jp \\ ${ }^{2}$ Department of Mathematics, Faculty of Science, Okayama University, Okayama 700-8530, Japan, \\ e-mail: hirokawa@math.okayama-u.ac.jp
}

\begin{abstract}
We consider a model of a quantum mechanical system coupled to a (massless) Bose field, called the generalized spin-boson model (A. Arai and M. Hirokawa, J. Funct. Anal. 151 (1997), 455-503), without infrared regularity condition. We define a regularized Hamiltonian $H(\nu)$ with a parameter $\nu \geq 0$ such that $H=H(0)$ is the Hamiltonian of the original model. We clarify a relation between ground states of $H(\nu)$ and those of $H$ by formulating sufficient conditions under which weak limits, as $\nu \rightarrow 0$, of the ground states of $H(\nu)$ 's are those of $H$. We also establish existence theorems on ground states of $H(\nu)$ and $H$ under weaker conditions than in the previous paper mentioned above.
\end{abstract}

Mathematics Subject Classifications (1991): 81Q10, 47B25, 47N50

Key Words: massless quantum field, Fock space, infrared problem, generalized spin-boson model, ground state, ground-state energy

\section{Contents}

1 Introduction 2

2 Some Fundamental Properties of the Model $\quad 6$

2.1 Self-adjointness . . . . . . . . . . . . . . . . . 6

2.2 Ground-state energy . . . . . . . . . . . . . . . . . . . 8

3 Analysis of the Regularized Hamiltonian 12

3.1 Strong resolvent convergence . . . . . . . . . . . . . . . . . . . . . 12

3.2 Ground-state energy . . . . . . . . . . . . . . . . . . . . 12

3.3 The ground-state expectation value of the number operator . . . . . . . . . 14

3.4 Upper bound for $n(\nu) \ldots \ldots \ldots \ldots \ldots \ldots$

3.5 Infrared divergence $\ldots \ldots \ldots \ldots \ldots \ldots$ 
4 Existence of Ground States of $H \quad 18$

4.1 Existence theorem . . . . . . . . . . . . . . . . . . . 18

4.2 Upper bound for $W(\nu) \ldots \ldots \ldots \ldots \ldots \ldots$

5 Existence of Ground States in the Regularized Theory 24

5.1 Preliminary results . . . . . . . . . . . . . . . . 24

5.2 Main result . . . . . . . . . . . . . . . . . . . 27

5.3 Existence of a ground state in the case $\mu=0$ with infrared regularity condition . . . . . . . . . . . . . . . . . . . 29

6 Examples 29

6.1 The van Hove Model . . . . . . . . . . . . . . . . . . . . . . . . . . . . . . 29

6.2 A quantum harmonic oscillator coupled to a Bose field . . . . . . . . . . 31

6.3 The Wigner-Weisskopf model . . . . . . . . . . . . . . . . . . . . 38

A Some General Properties of the Ground-State Energy for a Class of Self-Adjoint Operators

B Abstract Results on Discrete Spectrum of a Self-Adjoint Operator

\section{Introduction}

This work is a continuation of the previous one [15], in which we discussed existence and uniqueness of ground states of a model which gives an abstract unification of some quantum field models of particles interacting with a Bose field (we call the model a generalized spin-boson (GSB) model). In this paper we consider the model under weaker conditions than in [15] and establish theorems on existence of ground states of the model, generalizing those of [15].

If the Bose field is massless, then the GSB model can be regarded as an abstract simplified version of models of nonrelativistic quantum electrodynamics $[3,9,17,18,20,31$, $32,33,34,39,40,45]$. By this reason, it is particularly important to treat the case where the Bose field is massless. In this case, however, one encounters the "infrared problem", a number of problems related to the so-called "infrared catastrophe," a situation where the total energy of bosons emitted at low frequency is finite, but the number of such bosons ("soft bosons") blows up (e.g., [36, Chapter 4, §4-1-2], [21]; [27, 28] for mathematically rigorous discussions). Conventionally or heuristically the infrared catastrophe suggests absence of ground states (or other eigenvectors) of the model under consideration in the Hilbert space of state vectors where the "bare" boson number operator is defined. It turns out that it is a very subtle problem whether or not ground states exist in the original Hilbert space where the unperturbed part of the Hamiltonian of the model is defined via the usual Fock representation of canonical commutation relations. This aspect was investigated in [16] in view of absence of ground states. 
In contrast to the paper [16], we analyze, in the present paper, mathematical structures for existence (in the original Hilbert space) of ground states of the massless model without infrared regularity condition (for the exact meaning, see the paragraph after Remark 1.2 below). We establish existence theorems of ground states in the massless theory in terms of quantities defined as the mass-zero limits of those in the massive (regularized) theory.

To outline the present paper in more detail, we first describe the model. Let $\mathcal{H}$ be a complex Hilbert space and $\mathcal{F}_{\mathrm{b}}$ the Boson Fock space over $L^{2}\left(\mathbf{R}^{d}\right)$ :

$$
\mathcal{F}_{\mathrm{b}}:=\bigoplus_{n=0}^{\infty} \otimes_{\mathrm{s}}^{n} L^{2}\left(\mathbf{R}^{d}\right)
$$

where $\otimes_{\mathrm{s}}^{n} L^{2}\left(\mathbf{R}^{d}\right)$ denotes the $n$-fold symmetric tensor product of $L^{2}\left(\mathbf{R}^{d}\right), d \geq 1$, with $\otimes_{\mathrm{s}}^{0} L^{2}\left(\mathbf{R}^{d}\right):=\mathbf{C}$. The Hilbert space of the quantum field model we consider is

$$
\mathcal{F}:=\mathcal{H} \otimes \mathcal{F}_{\mathrm{b}}
$$

Let $\omega: \mathbf{R}^{d} \rightarrow[0, \infty)$ be Borel measurable such that $0<\omega(k)<\infty$ for almost everywhere (a.e.) $k \in \mathbf{R}^{d}$ with respect to the $d$-dimensional Lebesgue measure and $\hat{\omega}$ be the multiplication operator by the function $\omega$, acting in $L^{2}\left(\mathbf{R}^{d}\right)$. We denote by $d \Gamma(\hat{\omega})$ the second quantization of $\hat{\omega}[42, \S$ X.7]. Let $A$ be a self-adjoint operator on $\mathcal{H}$ bounded from below. Then the unperturbed Hamiltonian of the model is defined by

$$
H_{0}:=A \otimes I+I \otimes d \Gamma(\hat{\omega})
$$

with domain $D\left(H_{0}\right)=D(A \otimes I) \cap D(I \otimes d \Gamma(\hat{\omega}))$, where $I$ denotes identity operator and $D(T)$ the domain of an operator $T$. The operator $H_{0}$ is self-adjoint and bounded from below.

We denote by $a(f), f \in L^{2}\left(\mathbf{R}^{d}\right)$, the smeared annihilation operators on $\mathcal{F}_{\mathrm{b}}[a(f)$ is antilinear in $f][42, \S \mathrm{X} .7]$. Let $\lambda_{j} \in L^{2}\left(\mathbf{R}^{d}\right), j=1, \cdots, J$, with $J \in \mathbf{N}, B_{j}$ a closed linear operator on $\mathcal{H}$ and

$$
H_{I}:=\sum_{j=1}^{J}\left(B_{j} \otimes a\left(\lambda_{j}\right)^{*}+B_{j}^{*} \otimes a\left(\lambda_{j}\right)\right) .
$$

Let $\alpha \in \mathbf{R} \backslash\{0\}$ be a constant. Then the total Hamiltonian $H$ of the model is defined by

$$
H:=H_{0}+\alpha H_{I}
$$

acting in $\mathcal{F}$.

Remark 1.1 Davies [22] also treats a Hamiltonian of the same form as that of $H$, but, with $B_{j}$ bounded. We do not assume that each $B_{j}$ is bounded. Hence our Hamiltonian $H$ is a generalization of Davies'. 
Remark 1.2 If each $B_{j}$ is symmetric (i.e., $B_{j} \subset B_{j}^{*}$ ), then $H$ is the Hamiltonian of the $G S B$ model in [15]. But, in fact, even if each $B_{j}$ is not symmetric, $H$ can be written as a form of the Hamiltonian of the GSB model in [15] (see (2.15) in Section 2). Thus the class of the above $H$ coincides with that of the GSB model in [15]. A reason we use the form (1.4) with (1.3) is just that it is notationally convenient in dicussing examples we give in this paper (see Section 6).

Let

$$
\mu:=\operatorname{ess}_{\operatorname{sinf}} \mathbf{R}^{d} \omega(k),
$$

where ess.inf means essential infimum. We say that the model has low-energy cutoff if $\mu>0$. If the Bose field is massless with $\omega(k)=|k|$, then the model has no low-energy cutoff. Thus we are primarily interested in the model without low-energy cutoff. In this case, the behavior of the momentum-cutoff functions $\lambda_{j}(j=1, \cdots, J)$ in the neighborhood of $\omega(k)=0$ becomes significant. To explain this point briefly, we introduce a neighboring set

$$
D_{0}:=\left\{k \in \mathbf{R}^{d} \mid \omega(k) \leq 1\right\}
$$

of $\omega(k)=0$. For a set $S$, we denote by $\chi_{S}$ the characteristic function of $S$. If

$$
\int_{D_{0}} \frac{\left|\lambda_{j}(k)\right|^{2}}{\omega(k)^{2}} d k=+\infty, \quad j=1, \cdots, J
$$

i.e., $\lambda_{j} \chi_{D_{0}} / \omega \notin L^{2}\left(\mathbf{R}^{d}\right), j=1, \cdots, J$, then the model is said to have infrared singularity $[1,40,44]$. On the other hand, we say that the model is infraredly regular if $\lambda_{j} \chi_{D_{0}} / \omega \in$ $L^{2}\left(\mathbf{R}^{d}\right), j=1, \cdots, J$. Obviously the model with low-energy cutoff is infraredly regular (but the converse is not true). In the present paper we do not assume the infrared regularity for the model, otherwise stated.

A conventional picture is that the infrared singularity condition plus some condition gives absence of ground states of $H$ in $\mathcal{F}$. But this is a subtle problem as we remarked above. Indeed, there are some models which can have ground states even if the infrared singularity condition is fulfilled (e.g., $[4,5,6,7,10,11,12]$ )(for simple extreme examples, see Section 6 in the present paper), see also [18, §II].

In Section 2 we first prove self-adjointness of $H$ under some conditions. Then we estimate the ground-state energy of $H$ from below and above.

We want to analyze the model without infrared regularity from a model with infrared regularity. We call the latter an infraredly regularized model if it approximates the former in a suitable sense. A simple way to define such a regularized model is to replace $\omega$ by

$$
\omega_{\nu}(k):=\omega(k)+\nu
$$

with a constant $\nu>0$. The parameter $\nu$ plays a role of low-energy cutoff. It is obvious that $\lambda_{j} \chi_{D_{0}} / \omega_{\nu}^{s} \in L^{2}\left(\mathbf{R}^{d}\right)$ for all $s \geq 0$ and $j=1, \cdots, J$, and $\omega_{\nu}(k) \rightarrow \omega(k)(\nu \downarrow 0)$ for a.e. $k \in \mathbf{R}^{d}$. Hence the operator

$$
H(\nu):=A \otimes I+I \otimes d \Gamma\left(\hat{\omega}_{\nu}\right)+\alpha H_{I}
$$


may give a Hamiltonian of an infraredly regularized model. We have

$$
H(\nu)=H+\nu I \otimes N_{\mathrm{b}},
$$

where

$$
N_{\mathrm{b}}:=d \Gamma(I)
$$

is the number operator on $\mathcal{F}_{\mathrm{b}}$. This means that $H(\nu)$ can be viewed as an operator obtained as the perturbation of $H$ by the operator $\nu I \otimes N_{\mathrm{b}}$. Note that

$$
D(H(\nu))=D\left(H_{0}\right) \cap D\left(I \otimes N_{\mathrm{b}}\right), \quad \nu>0 .
$$

Remark 1.3 Of course there are other ways of regularizing the model $H$. For example, one may replace each $\lambda_{j}$ by $\lambda_{j}^{(\nu)}:=\chi_{\left\{k \in \mathbf{R}^{d} \mid \omega(k) \geq \nu\right\}} \lambda_{j}$. In this case $\nu$ is a parameter of infrared cutoff for boson momenta. If $\nu>0$, then we have $\lambda_{j}^{(\nu)} / \omega^{s} \in L^{2}\left(\mathbf{R}^{d}\right)$ for all $s>0$. Let $H(\nu)^{\prime}$ be the Hamiltonian $H$ with $\lambda_{j}$ replaced by $\lambda_{j}^{(\nu)}$. Then we can show that $H(\nu)^{\prime}$ converges to $H$ in the norm resolvent sense as $\nu \rightarrow 0$ (the proof is similar to that of [15, Lemma 3.5]). On the other hand, as we shall see below (Proposition 3.1), $H(\nu)$ converges to $H$ in the strong resolvent sense as $\nu \rightarrow 0$. In this respect, the regularization using $H(\nu)^{\prime}$ is stronger than ours using $H(\nu)$. However it turns out that the former regularization is inconvenient to fromulate conditions for the existence of a ground state of $H$ in terms of the behavior of the ground-state energy of a regularized Hamiltonian, as the regularization is removed.

In Section 3 we analyze the regularized Hamiltonian $H(\nu)$ including the case $\nu=0$ with the following aspects: (i) the ground-state energy $E_{0}(\nu)$ of $H(\nu)$ as a function of $\nu \in[0, \infty)$; (ii) the ground-state expectation value $n(\nu)$ of the number operator $I \otimes N_{\mathrm{b}}$ for $\nu>0$ under the assumption of existence of a ground-state of $H(\nu)$; (iii) the infrared divergence in the sense that $n(\nu) \rightarrow \infty$ as $\nu \rightarrow 0$.

Section 4 is devoted to a structural analysis on conditions for $H$ to have a ground state as a subsequence weak limit of ground states of $H(\nu)$ 's as $\nu \rightarrow 0$. The conditions are described in terms of the behavior of $n(\nu)$ and a correlation function $W(\nu)$ [see (4.5)] as $\nu \rightarrow 0$ or $E_{0}^{\prime}(0+)$, the right differential of $E_{0}(\nu)$ at $\nu=0$. The analysis clarifies a relation between ground states of $H(\nu)$ and those of $H$. We also estimate an upper bound for the correlation function $W(\nu)$. We regard the results of this section as some of most important results of the present paper.

Remark 1.4 Experimentally a quantum state is identified through observations of observables which give numerical quantities such as energy levels, the mean boson number, and some correlation functions etc. Thus, from a physical point of view based on this picture, characterizing existence of ground states in terms of experimentally observed numerical functions are natural. 
In Section 5 we prove existence of ground states of the Hamiltonian with low-energy cutoff, which includes the regularized one $H(\nu)$ as a special case, and the Hamiltonian with infrared regularity.

As for the existence of ground states of $H$ and $H(\nu)$, the previous paper [15] assumed that the spectrum of $A$ is purely discrete. But, in the present work, we do not assume it. This is an important point which improves the existence results of ground states in [15].

In the last section, we discuss some simple examples (the van Hove model, a model of a quantum harmonic oscillator coupled to a Bose field with a rotating wave approximation and the Wigner-Weisskopf model) in view of the analysis of Section 4. The van Hove model is an example which has no ground states if the infrared singularity condition is fulfilled. On the other hand, the other two models are examples which can have ground states even if the infrared singularity condition is fulfilled. It is instructive to see, in each example, the behavior of the derivative of the ground-state energy of the regularized model as $\nu \rightarrow 0$.

The present paper has two appendices. Appendix A presents some results on the ground-state energy for a class of self-adjoint operators on the abstract Hilbert space. These results are applied to the ground-state energy $E_{0}(\nu)$ of $H(\nu)$. In Appendix B we establish a general perturbation theorem on discrete spectrum of a self-adjoint operator. This is applied to proof of existence of ground states of the Hamiltonian with low-energy cutoff(Section 5).

Remark 1.5 A general treatment of spectral-theoretical aspects of models of GSB's type has been made by Dereziński and Jakšić [24]. An analysis on essential spectrum is given in [14].

\section{Some Fundamental Properties of the Model}

\subsection{Self-adjointness}

The inner product (resp. norm) of a Hilbert space $\mathcal{K}$ is denoted $(\cdot, \cdot)_{\mathcal{K}}$, complex linear in the second variable (resp. $\|\cdot\|_{\mathcal{K}}$ ). But, if there is no danger of confusion, then we omit the subscript $\mathcal{K}$ in $(\cdot, \cdot)_{\mathcal{K}}$ and $\|\cdot\|_{\mathcal{K}}$.

For each $s \in \mathbf{R}$, we define a Hilbert space

$$
\mathcal{M}_{s}=\left\{f: \mathbf{R}^{d} \rightarrow \mathbf{C} \text {, Borel measurable } \mid \omega^{s / 2} f \in L^{2}\left(\mathbf{R}^{d}\right)\right\}
$$

with inner product $(f, g)_{s}:=\left(\omega^{s / 2} f, \omega^{s / 2} g\right)_{L^{2}\left(\mathbf{R}^{d}\right)}$ and norm

$$
\|f\|_{s}:=\left\|\omega^{s / 2} f\right\|_{L^{2}\left(\mathbf{R}^{d}\right)}, \quad f \in \mathcal{M}_{s} .
$$

For a linear operator $T$, we denote its spectrum by $\sigma(T)$. If $T$ is a self-adjoint operator bounded from below, then we define

$$
\mathcal{E}_{0}(T):=\inf \sigma(T),
$$


the ground-state energy of $T$.

We define

$$
\widetilde{A}:=A-\mathcal{E}_{0}(A),
$$

which is a nonnegative self-adjoint operator.

The basic hypothesises for the model are the following (H.1) and (H.2):

(H.1) $\lambda_{j} \in \mathcal{M}_{-1} \cap \mathcal{M}_{0}, \quad j=1, \cdots, J$.

(H.2) $D\left(\widetilde{A}^{1 / 2}\right) \subset D\left(B_{j}\right) \cap D\left(B_{j}^{*}\right), j=1, \cdots, J$, and there exist constants $a_{j, \pm} \geq 0$, $b_{j, \pm} \geq 0, j=1, \cdots, J$, such that, for all $u \in D\left(\widetilde{A}^{1 / 2}\right)$,

$$
\left\|\frac{\left(B_{j}^{*} \pm B_{j}\right)}{\sqrt{2}} u\right\|^{2} \leq a_{j, \pm}^{2}\left\|\widetilde{A}^{1 / 2} u\right\|^{2}+b_{j, \pm}^{2}\|u\|^{2}, \quad j=1, \cdots, J,
$$

and

$$
|\alpha|\left(\sum_{j=1}^{J}\left(a_{j,+}+a_{j,-}\right)\left\|\lambda_{j}\right\|_{-1}\right)<1
$$

Assume (H.2) and set

$$
a_{j}:=\sqrt{a_{j,+}^{2}+a_{j,-}^{2}}, \quad b_{j}:=\sqrt{b_{j,+}^{2}+b_{j,-}^{2}}, \quad j=1, \cdots, J .
$$

Then, by the identity (the parallelogram law)

$$
\left\|B_{j} u\right\|^{2}+\left\|B_{j}^{*} u\right\|^{2}=\left\|\frac{B_{j}^{*}+B_{j}}{\sqrt{2}} u\right\|^{2}+\left\|\frac{B_{j}^{*}-B_{j}}{\sqrt{2}} u\right\|^{2},
$$

we have for all $u \in D\left(\widetilde{A}^{1 / 2}\right)$,

$$
\begin{gathered}
\left\|B_{j} u\right\|^{2}+\left\|B_{j}^{*} u\right\|^{2} \leq a_{j}^{2}\left\|\widetilde{A}^{1 / 2} u\right\|^{2}+b_{j}^{2}\|u\|^{2}, \\
j=1, \cdots, J,
\end{gathered}
$$

For a vector $v=\left(v_{j}\right)_{j=1}^{J} \in \mathbf{R}^{J}$ and $f=\left(f_{j}\right)_{j=1}^{J} \in \oplus^{J} L^{2}\left(\mathbf{R}^{d}\right)$, we define

$$
M_{v}(f):=\sum_{j=1}^{J} v_{j}\left\|f_{j}\right\|_{L^{2}\left(\mathbf{R}^{d}\right)}
$$

We set

$$
v(a):=\left(a_{j,+}+a_{j,-}\right)_{j=1}^{J}, \quad v(b):=\left(b_{j,+}+b_{j,-}\right)_{j=1}^{J} .
$$

For positive constants $\varepsilon, \varepsilon^{\prime}$, we introduce

$$
\begin{aligned}
& F_{\varepsilon, \varepsilon^{\prime}}^{(a, b)}(\lambda, \omega):=M_{v(a)}(\lambda / \sqrt{\omega})+\frac{\varepsilon}{\sqrt{2}} M_{v(a)}(\lambda)+\sqrt{2} \varepsilon^{\prime} M_{v(b)}(\lambda / \sqrt{\omega}), \\
& G_{\varepsilon, \varepsilon^{\prime}}^{(a, b)}(\lambda, \omega):=\frac{1}{4 \sqrt{2} \varepsilon} M_{v(a)}(\lambda)+\frac{1}{2 \sqrt{2} \varepsilon^{\prime}} M_{v(b)}(\lambda / \sqrt{\omega})+\frac{M_{v(b)}(\lambda)}{\sqrt{2}},
\end{aligned}
$$

We define

$$
\widetilde{H}_{0}:=H_{0}-\mathcal{E}_{0}(A)=\widetilde{A} \otimes I+I \otimes d \Gamma(\hat{\omega}) \geq 0 .
$$


Proposition 2.1 Assume (H.1) and (H.2). Then $D\left(H_{0}\right) \subset D\left(H_{I}\right), H$ is self-adjoint on $D(H)=D\left(H_{0}\right)$ and bounded from below. Moreover, $H$ is essentially self-adjoint on every core of $H_{0}$.

Proof. Using (2.6), we can show in the same way as in the proof of [15, Proposition 1.1] that $B_{j} \otimes a\left(\lambda_{j}\right)^{*}$ and $B_{j}^{*} \otimes a\left(\lambda_{j}\right)$ are $H_{0}$-bounded, so that $D\left(H_{0}\right) \subset D\left(H_{I}\right)$. Let

$$
\phi(f):=\frac{a(f)^{*}+a(f)}{\sqrt{2}}, \quad f \in L^{2}\left(\mathbf{R}^{d}\right)
$$

and

$$
\begin{aligned}
S_{j} & :=\frac{B_{j}+B_{j}^{*}}{\sqrt{2}}, \quad S_{J+j}:=\frac{i\left(B_{j}^{*}-B_{j}\right)}{\sqrt{2}}, \\
g_{j} & :=\lambda_{j}, \quad g_{J+j}:=i \lambda_{j}, \quad j=1, \cdots, J .
\end{aligned}
$$

Then we have

$$
H_{I}=\sum_{j=1}^{2 J} S_{j} \otimes \phi\left(g_{j}\right) .
$$

Hence $H$ is of the form of the Hamiltonian of the GSB model introduced in [15]. Hence we can apply the estimate (2.9) in [15] to obtain the following inequality:

$$
\left\|H_{I} \Psi\right\|_{\mathcal{F}} \leq F_{\varepsilon, \varepsilon^{\prime}}^{(a, b)}(\lambda, \omega)\left\|\widetilde{H_{0}} \Psi\right\|_{\mathcal{F}}+G_{\varepsilon, \varepsilon^{\prime}}^{(a, b)}(\lambda, \omega)\|\Psi\|_{\mathcal{F}}, \Psi \in D\left(H_{0}\right) .
$$

Condition (2.4) implies that $|\alpha| F_{\varepsilon, \varepsilon^{\prime}}^{(a, b)}(\lambda, \omega)<1$ for all sufficiently small $\varepsilon$ and $\varepsilon^{\prime}$. Hence, by the Kato-Rellich theorem, we obtain the desired results.

\subsection{Ground-state energy}

For a self-adjoint operator $T$ on a Hilbert space $\mathcal{X}$, we denote by $P_{T}$ its spectral measure and by $Q(T)$ its form domain: $Q(T):=D\left(|T|^{1 / 2}\right)$. The sesquilinear form $q_{T}$ associated with $T$ is deifned by

$$
q_{T}(\psi, \phi):=\int_{\mathbf{R}} \lambda d\left(\psi, P_{T}(\lambda) \phi\right)_{\mathcal{X}}, \quad \psi, \phi \in Q(T)
$$

Assume (H.1) and (H.2). Then we can define a sesquilinear form $q(u, v)$ with form domain $Q(q):=Q(A)$ by

$$
q(u, v):=q_{A}(u, v)-\sum_{j=1}^{J} \alpha^{2}\left\|\lambda_{j}\right\|_{-1}^{2}\left(B_{j} u, B_{j} v\right)_{\mathcal{H}}, \quad u, v \in Q(q) .
$$


Lemma 2.2 Assume (H.1) and (H.2). Then there exists a unique self-adjoint operator $L$ on $\mathcal{H}$ such that $Q(L)=Q(q)$ and

$$
q_{L}(u, v)=q(u, v), \quad u, v \in Q(L) .
$$

Moreover, $L$ is bounded from below with

$$
L \geq \mathcal{E}_{0}(A)-\sum_{j=1}^{J} \alpha^{2} b_{j}^{2}\left\|\lambda_{j}\right\|_{-1}^{2}
$$

and every core of $A$ is a form core of $L$.

Proof. By (2.6), we have

$$
\sum_{j=1}^{J} \alpha^{2}\left\|\lambda_{j}\right\|_{-1}^{2}\left\|B_{j} u\right\|_{\mathcal{H}}^{2} \leq\left(\sum_{j=1}^{J} \alpha^{2} a_{j}^{2}\left\|\lambda_{j}\right\|_{-1}^{2}\right)\left\|\widetilde{A}^{1 / 2} u\right\|_{\mathcal{H}}^{2}+\left(\sum_{j=1}^{J} \alpha^{2} b_{j}^{2}\left\|\lambda_{j}\right\|_{-1}^{2}\right)\|u\|_{\mathcal{H}}^{2} .
$$

By (2.4),

$$
\sum_{j=1}^{J} \alpha^{2} a_{j}^{2}\left\|\lambda_{j}\right\|_{-1}^{2} \leq \alpha^{2}\left(\sum_{j=1}^{J}\left(a_{j,+}+a_{j,-}\right)\left\|\lambda_{j}\right\|_{-1}\right)^{2}<1 .
$$

Hence, by the KLMN theorem [42, Theorem X.17], there exists a unique self-adjoint operator $\hat{L}$ with $Q(\hat{L})=D\left(\widetilde{A}^{1 / 2}\right)=Q(q)$ such that

$$
q_{\hat{L}}(u, v)=\left\|\widetilde{A}^{1 / 2} u\right\|_{\mathcal{H}}^{2}-\sum_{j=1}^{J} \alpha^{2}\left\|\lambda_{j}\right\|_{-1}^{2}\left\|B_{j} u\right\|_{\mathcal{H}}^{2}=q(u, v)-\mathcal{E}_{0}(A)
$$

and $\hat{L} \geq-\sum_{j=1}^{J} \alpha^{2} b_{j}^{2}\left\|\lambda_{j}\right\|_{-1}^{2}$. Putting $L:=\hat{L}+\mathcal{E}_{0}(A)$, we obtain the desired results.

A lower bound for the ground-state energy $\mathcal{E}_{0}(H)$ of $H$ is given in the following proposition.

Proposition 2.3 Assume (H.1) and (H.2). Then

$$
H \geq L \otimes I
$$

in the sense of sesquilinear form. In particular,

$$
\mathcal{E}_{0}(L) \leq \mathcal{E}_{0}(H)
$$

and

$$
\mathcal{E}_{0}(A)-\sum_{j=1}^{J} \alpha^{2} b_{j}^{2}\left\|\lambda_{j}\right\|_{-1}^{2} \leq \mathcal{E}_{0}(H)
$$


Proof. Let $\mathcal{F}_{\mathrm{b}, 0}$ be the subspace of finite-particle vectors in $\mathcal{F}_{\mathrm{b}}$ :

$$
\mathcal{F}_{\mathrm{b}, 0}:=\left\{\psi=\left\{\psi^{(n)}\right\}_{n=0}^{\infty} \in \mathcal{F}_{\mathrm{b}} \mid \psi^{(n)}=0 \text { for all but finitely many } n \text { 's }\right\}
$$

and

$$
D_{0, \infty}:=\left\{\psi \in \mathcal{F}_{\mathrm{b}, 0} \mid \psi^{(n)} \in C_{0}^{\infty}\left(\mathbf{R}^{d n}\right) \text { for all } n \geq 1\right\},
$$

where $C_{0}^{\infty}\left(\mathbf{R}^{d n}\right)$ is the space of infinitely differentiable functions on $\mathbf{R}^{d n}$ with compact support. Then, for each $k \in \mathbf{R}^{d}$, we can define a linear operator $a(k)$ on $\mathcal{F}_{\mathbf{b}}$ with $D(a(k))=$ $D_{0, \infty}$ by

$$
(a(k) \psi)^{(n)}\left(k_{1}, \cdots, k_{n}\right):=\sqrt{n+1} \psi^{(n+1)}\left(k, k_{1}, \cdots, k_{n}\right),
$$

for a.e. $\left(k_{1}, \cdots, k_{n}\right) \in \mathbf{R}^{\nu n}$ (cf. [42, §X.7]). For all $f \in L^{2}\left(\mathbf{R}^{d}\right)$ and $\psi \in D_{0, \infty}$, we have

$$
a(f) \psi=\int_{\mathbf{R}^{d}} f(k)^{*} a(k) \psi d k,
$$

where the integral is taken in the sense of $\mathcal{F}_{\mathrm{b}}$-valued strong Bochner integral.

We denote by $L_{\text {loc }}^{2}\left(\mathbf{R}^{d}\right)$ the space of Borel measurable functions $f$ on $\mathbf{R}^{d}$ such that, for all $R>0, \int_{|k| \leq R}|f(k)|^{2} d k<\infty$. We first consider the case $\omega \in L_{\text {loc }}^{2}\left(\mathbf{R}^{d}\right)$. Then

$$
(\psi, d \Gamma(\hat{\omega}) \psi)=\int_{\mathbf{R}^{d}} \omega(k)\|a(k) \psi\|^{2} d k, \quad \psi \in D_{0, \infty} .
$$

Let $\Psi \in D(A) \otimes_{\text {alg }} D_{0, \infty}$, where $\otimes_{\text {alg }}$ means algebraic tensor product. Then we have

$$
(\Psi, H \Psi)=q_{L \otimes I}(\Psi, \Psi)+\int_{\mathbf{R}^{d}} \omega(k)\left\|\left(I \otimes a(k)+\alpha \lambda_{j}(k) \omega(k)^{-1} B_{j} \otimes I\right) \Psi\right\|^{2} d k .
$$

Since the second term on the right hand side is nonnegative, we obtain

$$
(\Psi, H \Psi) \geq q_{L \otimes I}(\Psi, \Psi) .
$$

The condition $\omega \in L_{\text {loc }}^{2}\left(\mathbf{R}^{d}\right)$ implies that $D_{0, \infty}$ is a core of $d \Gamma(\hat{\omega})$. Hence $D(A) \otimes_{\text {alg }} D_{0, \infty}$ is a core of $H_{0}$. Hence, by Proposition $2.1, D(A) \otimes_{\text {alg }} D_{0, \infty}$ is a core of $H$. Thus $(2.22)$ extends to all $\Psi \in D(H)=D\left(H_{0}\right)$, implying (2.19).

We next consider the case where $\omega \notin L_{\mathrm{loc}}^{2}\left(\mathbf{R}^{d}\right)$. We define $\omega^{(n)}:=\left(1+n^{-1} \omega\right)^{-1} \omega, n \geq 1$, and denote by $H_{n}$ (resp. $L_{n}$ ) the operator $H$ (resp. $L$ ) with $\omega$ replaced by $\omega^{(n)}$. We have $\omega^{(n)} \in L_{\text {loc }}^{2}\left(\mathbf{R}^{d}\right), \lambda_{j} / \sqrt{\omega^{(n)}} \in L^{2}\left(\mathbf{R}^{d}\right)$, and $\omega^{(n)} \leq \omega, \omega^{(n)}(k) \uparrow \omega(k)(n \rightarrow \infty)$ for a.e. $k$. By the preceding result, we have $q_{L_{n} \otimes I}(\Psi, \Psi) \leq\left(\Psi, H_{n} \Psi\right), \Psi \in D\left(H_{0}\right)$. It is easy to see that, for all $\Psi \in D\left(H_{0}\right), q_{L_{n} \otimes I}(\Psi, \Psi) \rightarrow q_{L \otimes I}(\Psi, \Psi),\left\|H_{n} \Psi-H \Psi\right\| \rightarrow 0$ as $n \rightarrow \infty$. Hence $q_{L \otimes I}(\Psi, \Psi) \leq(\Psi, H \Psi), \Psi \in D\left(H_{0}\right)$, which implies $(2.19)$.

Inequality (2.20) follows from (2.19) and the variational principle. Estimate (2.21) is obtained from (2.20) and Lemma 2.2.

We next consider upper bounds of $\mathcal{E}_{0}(H)$. For this purpose, we define a nonlinear functional $F$ on $D(A)$ by

$$
F(u):=(u, A u)-\alpha^{2}\left\|\frac{\sum_{j=1}^{J}\left(u, B_{j} u\right) \lambda_{j}}{\sqrt{\omega}}\right\|_{L^{2}\left(\mathbf{R}^{d}\right)}^{2}, \quad u \in D(A),
$$


and set

$$
F_{0}:=\inf _{u \in D(A) ;\|u\|=1} F(u) .
$$

Proposition 2.4 Assume (H.1) and (H.2). Then

$$
\mathcal{E}_{0}(H) \leq F_{0} .
$$

Proof. Let $u \in D(A)$ and $\psi \in D(d \Gamma(\hat{\omega}))$ with $\|u\|=1=\|\psi\|$. Then $\|u \otimes \psi\|=1$ and we have

$$
(u \otimes \psi, H u \otimes \psi)=(u, A u)+\left(\psi, H_{\mathrm{VH}}(g) \psi\right)
$$

where

$$
H_{\mathrm{VH}}(g):=d \Gamma(\omega)+\alpha \phi(g)
$$

with $g:=\sum_{j=1}^{J} \sqrt{2}\left(u, B_{j} u\right) \lambda_{j}$. By the variational principle, we have $\mathcal{E}_{0}(H) \leq(u \otimes \psi, H u \otimes$ $\psi$ ). Hence

$$
\mathcal{E}_{0}(H) \leq(u, A u)+\left(\psi, H_{\mathrm{VH}}(g) \psi\right) .
$$

On the other hand, $H_{\mathrm{VH}}(g)$ is a Hamiltonian of the so-called van Hove model or a fixed source model (e.g., [25, Chaper 1, §e] and references therein). Properties of this model are well known, including the case without low-energy cutoff(e.g., [13]). In particular,

$$
\mathcal{E}_{0}\left(H_{\mathrm{VH}}(g)\right)=-\frac{\alpha^{2}}{2}\left\|\frac{g}{\sqrt{\omega}}\right\|_{L^{2}\left(\mathbf{R}^{d}\right)}^{2}=-\alpha^{2}\left\|\frac{\sum_{j=1}^{J}\left(u, B_{j} u\right) \lambda_{j}}{\sqrt{\omega}}\right\|_{L^{2}\left(\mathbf{R}^{d}\right)}^{2} .
$$

Thus (2.25) follows.

Remark 2.1 The operator $H_{\mathrm{VH}}(g)$ is a special case of $H$. Namely, if we take $\mathcal{H}=\mathbf{C}$, then the Hamiltonian $H$ with $A=0, J=1, B_{1}=1$ and $\lambda_{1}=g$ yields $H_{\mathrm{VH}}(g)$.

Remark 2.2 Since $F(u) \leq(u, A u), u \in D(A)$, we have

$$
F_{0} \leq \mathcal{E}_{0}(A) \text {. }
$$

Hence, by (2.25),

$$
\mathcal{E}_{0}(H) \leq \mathcal{E}_{0}(A) .
$$

Suppose, in addition to (H.1) and (H.2), that $A$ has a normalized ground state $u_{0}: A u_{0}=$ $\mathcal{E}_{0}(A) u_{0}$ such that, for some $j,\left(u_{0}, B_{j} u_{0}\right) \neq 0$ and that $\lambda_{1}, \cdots, \lambda_{J}$ are linearly independent. Then we have

$$
F\left(u_{0}\right)=\mathcal{E}_{0}(A)-\alpha^{2}\left\|\frac{\sum_{j=1}^{J}\left(u_{0}, B_{j} u_{0}\right) \lambda_{j}}{\sqrt{\omega}}\right\|_{L^{2}\left(\mathbf{R}^{d}\right)}^{2}<\mathcal{E}_{0}(A) .
$$

Hence, in this case,

$$
\mathcal{E}_{0}(H)<\mathcal{E}_{0}(A)
$$

i.e., the interaction makes the ground-state energy strictly lower.

Remark 2.3 A special choice of $\mathcal{H}, A$ and $B_{j}$ yields the standard spin-boson (SSB) model [15]. Some approximate expressions for the ground state energy of the SSB model are found in [29, 46]. Recently [30] gives an exact and explicit representation for that. 


\section{Analysis of the Regularized Hamiltonian}

Throughout this section, we assume (H.1) and (H.2).

\subsection{Strong resolvent convergence}

Let $H(\nu)$ be defined by (1.10). It is easy to see that (H.1) is satisfied with $\omega$ replaced by $\omega_{\nu}$. Hence Proposition 2.1 holds with $H$ replaced by $H(\nu)$.

We denote by

$$
\Omega:=\{1,0,0, \cdots\} \in \mathcal{F}_{\mathrm{b}}
$$

the Fock vacuum in $\mathcal{F}_{\mathrm{b}}$. We define

$$
\mathcal{F}_{\text {fin }}\left(\omega_{\nu}\right):=\mathcal{L}\left\{\Omega, a\left(f_{1}\right)^{*} \cdots a\left(f_{n}\right)^{*} \Omega \mid n \in \mathbf{N}, f_{j} \in D\left(\widehat{\omega}_{\nu}\right), j=1, \cdots, J\right\},
$$

where $\mathcal{L}\{\cdots\}$ denotes the subspace algebraically spaned by the vectors in the set $\{\cdots\}$. The subspace $\mathcal{F}_{\text {fin }}\left(\omega_{\nu}\right)$ is dense in $\mathcal{F}_{b}$ and a core for $d \Gamma\left(\widehat{\omega}_{\nu}\right)$. Note that $D(\widehat{\omega})=D\left(\widehat{\omega}_{\nu}\right)$, so that

$$
\mathcal{F}_{\text {fin }}(\omega)=\mathcal{F}_{\text {fin }}\left(\omega_{\nu}\right)
$$

We introduce a dense subspace $\mathcal{D}_{\omega}$ in $\mathcal{F}$ by

$$
\mathcal{D}_{\omega}:=D(A) \otimes_{\text {alg }} \mathcal{F}_{\text {fin }}(\omega) .
$$

Proposition 3.1 (i) $\mathcal{D}_{\omega}$ is a common core of $\{H(\nu)\}_{\nu \geq 0}$ and, for all $\Psi \in \mathcal{D}_{\omega}$,

$$
\lim _{\nu \rightarrow 0}\|H(\nu) \Psi-H \Psi\|=0 .
$$

(ii) For all $z \in \mathbf{C} \backslash \mathbf{R}$ and $\Psi \in \mathcal{F}$,

$$
\lim _{\nu \downarrow 0}\left\|(H(\nu)-z)^{-1} \Psi-(H-z)^{-1} \Psi\right\|=0 .
$$

Proof. (i) The subspace $\mathcal{D}_{\omega}$ is a core of $H_{0}$. Hence, Proposition 2.1, it is a core of $H(\nu)$ for all $\nu \geq 0$. Eq.(3.5) easily follows from the equation $H(\nu) \Psi-H \Psi=\nu I \otimes N_{\mathrm{b}} \Psi$.

(ii) This follows from part (i) and an application of a general convergence theorem [41, Theorem VIII.25 (a)].

\subsection{Ground-state energy}

For notational simplicity, we denote by $E_{0}(\nu)$ the ground-state energy of $H(\nu)$ :

$$
E_{0}(\nu):=\mathcal{E}_{0}(H(\nu))=\inf \sigma(H(\nu))
$$


Let $F^{(\nu)}$ is the functional $F$ with $\omega$ replaced by $\omega_{\nu}$ :

$$
F^{(\nu)}(u):=(u, A u)-\alpha^{2}\left\|\frac{\sum_{j=1}^{J}\left(u, B_{j} u\right) \lambda_{j}}{\sqrt{\omega_{\nu}}}\right\|_{L^{2}\left(\mathbf{R}^{d}\right)}^{2}
$$

and set

$$
F_{0}(\nu):=\inf _{u \in D(A) ;\|u\|=1} F^{(\nu)}(u) .
$$

Then, by Propositions 2.3 and 2.4, we have

$$
\mathcal{E}_{0}(A)-\sum_{j=1}^{J} \alpha^{2} b_{j}^{2}\left\|\frac{\lambda_{j}}{\sqrt{\omega_{\nu}}}\right\|_{L^{2}\left(\mathbf{R}^{d}\right)}^{2} \leq E_{0}(\nu) \leq F_{0}(\nu) \leq \mathcal{E}_{0}(A) .
$$

Basic analytical properties of $E_{0}(\nu)$ as a function of $\nu \geq 0$ are summarized in the following proposition.

Proposition 3.2 (i) The function $E_{0}(\nu)$ is monotone nondecreasing in $\nu \geq 0$.

(ii) The function $E_{0}(\nu)$ is concave, i.e., for all $\nu, \nu^{\prime} \in[0, \infty)$ and $t \in[0,1]$,

$$
t E_{0}(\nu)+(1-t) E_{0}\left(\nu^{\prime}\right) \leq E_{0}\left(t \nu+(1-t) \nu^{\prime}\right)
$$

(iii) The function $E_{0}(\nu)$ is continuous on $[0, \infty)$. In particular,

$$
\lim _{\nu \downarrow 0} E_{0}(\nu)=E_{0}(0) .
$$

(iv) For all $\nu>0$,

$$
E_{0}^{\prime}(\nu \pm 0):=\lim _{\varepsilon \downarrow 0} \frac{E_{0}(\nu \pm \varepsilon)-E_{0}(\nu)}{ \pm \varepsilon}
$$

exist and

$$
E_{0}^{\prime}(\nu+0) \leq E_{0}^{\prime}(\nu-0)
$$

$(\mathrm{v})$

$$
\lim _{\nu \rightarrow \infty} E_{0}(\nu)=\mathcal{E}_{0}(A)
$$

Proof. Parts (i)-(iv) follow from a simple application of Proposition A.1 in Appendix A with $T=H$ and $S=I \otimes N_{\mathrm{b}}$ (note that $D(H) \cap D\left(I \otimes N_{\mathrm{b}}\right)=D\left(H_{0}\right) \cap D\left(I \otimes N_{\mathrm{b}}\right)$ is a core of $H_{0}$ and hence of $H$ ). As for part (iv), we first note that

$$
\lim _{\nu \rightarrow \infty}\left\|\frac{\lambda_{j}}{\sqrt{\omega_{\nu}}}\right\|_{L^{2}\left(\mathbf{R}^{d}\right)}^{2}=0
$$


and, for all $u \in D(A)$,

$$
\lim _{\nu \rightarrow \infty}\left\|\frac{\sum_{j=1}^{J}\left(u, B_{j} u\right) \lambda_{j}}{\sqrt{\omega_{\nu}}}\right\|_{L^{2}\left(\mathbf{R}^{d}\right)}^{2}=0 .
$$

Hence, by (3.10),

$$
\mathcal{E}_{0}(A) \leq \liminf _{\nu \rightarrow \infty} E_{0}(\nu) \leq \limsup _{\nu \rightarrow \infty} E_{0}(\nu) \leq(u, A u)
$$

for all $u \in D(A)$ with $\|u\|=1$. Since $\mathcal{E}_{0}(A)=\inf _{u \in D(A) ;\|u\|=1}(u, A u)$, (3.15) follows.

\subsection{The ground-state expectation value of the number operator}

In what follows, we assume the following in addition to (H.1) and (H.2):

(H.3) There exists a constant $\nu_{0}>0$ such that, for all $\nu \in\left(0, \nu_{0}\right), H(\nu)$ has a ground state $\Psi_{0}(\nu)$ with $\left\|\Psi_{0}(\nu)\right\|=1$.

For a linear operator $X$ on $\mathcal{F}$ with $D(X) \supset D\left(H_{0}\right) \cap D\left(I \otimes N_{\mathrm{b}}\right)$, we define

$$
\langle X\rangle_{\nu}:=\left(\Psi_{0}(\nu), X \Psi_{0}(\nu)\right), \quad \nu \in\left(0, \nu_{0}\right),
$$

the ground-state expectation value of $X$. We set

$$
n(\nu):=\left\langle I \otimes N_{\mathrm{b}}\right\rangle_{\nu}, \quad \nu \in\left(0, \nu_{0}\right),
$$

and define

$$
\begin{aligned}
\bar{n} & :=\limsup _{\nu \downarrow 0} n(\nu), \\
\underline{n} & :=\liminf _{\nu \downarrow 0} n(\nu) .
\end{aligned}
$$

Proposition 3.3 (i) $\lim _{\nu \downarrow 0} \nu \cdot n(\nu)=0$.

(ii) $\lim _{\nu \downarrow 0}\langle H\rangle_{\nu}=E_{0}(0)$.

(iii) For all $\nu \in\left(0, \nu_{0}\right)$,

$$
n(\nu) \geq E_{0}^{\prime}(\nu+0) .
$$

In particular,

$$
\underline{n} \geq \liminf _{\nu \downarrow 0} E_{0}^{\prime}(\nu+0), \quad \bar{n} \geq \limsup _{\nu \downarrow 0} E_{0}^{\prime}(\nu+0) .
$$

(iv) If the right differential

$$
E_{0}^{\prime}(0+):=\lim _{\nu \downarrow 0} \frac{E_{0}(\nu)-E_{0}(0)}{\nu}
$$

of $E_{0}(\nu)$ at $\nu=0$ exists, then

$$
\bar{n} \leq E_{0}^{\prime}(0+)
$$


(v) $\bar{n}<\infty$ if and only if $E_{0}(\nu)-\langle H\rangle_{\nu}=O(\nu)(\nu \downarrow 0)$, where $O(\cdot)$ is Landau's symbol.

Proof. We need only apply Proposition A.3 in Appendix A with $T=H$ and $S=I \otimes N_{\mathrm{b}}$.

\subsection{Upper bound for $n(\nu)$}

We define

$$
R_{j}(\nu):=\left\|B_{j} \otimes I \Psi_{0}(\nu)\right\|^{2} .
$$

Note that, if $\Psi_{0}(\nu) \in D\left(\left(B_{j}^{*} \otimes I\right)\left(B_{j} \otimes I\right)\right)$, then $R_{j}(\nu)=\left(\Psi_{0}(\nu),\left(B_{j}^{*} \otimes I\right)\left(B_{j} \otimes I\right) \Psi_{0}(\nu)\right)$, i.e., $R_{j}(\nu)$ is the ground-state expectation value of $\left(B_{j}^{*} \otimes I\right)\left(B_{j} \otimes I\right)$ or the correlation function of $\left(B_{j}^{*} \otimes I\right)$ and $\left(B_{j} \otimes I\right)$ in the ground state $\Psi_{0}(\nu)$.

Proposition 3.4 For all $\nu \in\left(0, \nu_{0}\right)$,

$$
n(\nu) \leq \alpha^{2}\left(\sum_{j=1}^{J}\left\|\frac{\lambda_{j}}{\omega_{\nu}}\right\| \sqrt{R_{j}(\nu)}\right)^{2} .
$$

Proof. Similar to the proof of [15, Lemma 4.3].

This proposition immediately yields the following fact:

Corollary 3.5 Suppose that, for each $j=1, \cdots, J$,

$$
K_{j}:=\limsup _{\nu \downarrow 0}\left\|\frac{\lambda_{j}}{\omega_{\nu}}\right\| \sqrt{R_{j}(\nu)}
$$

is finite. Then

$$
\bar{n} \leq \alpha^{2}\left(\sum_{j=1}^{J} K_{j}\right)^{2}<\infty .
$$

Remark 3.1 Suppose that $\left\{\lambda_{1}, \cdots, \lambda_{J}, \omega\right\}$ obeys the infrared singularity condition (1.7). Then $\lim _{\nu \downarrow 0}\left\|\lambda_{j} / \omega_{\nu}\right\|=+\infty, j=1, \cdots, J$. But it is possible for the assumption of Corollary 3.5 to hold if

$$
\lim _{\nu \downarrow 0} R_{j}(\nu)=0, \quad j=1, \cdots, J .
$$

We can estimate $R_{j}(\nu)$ from above. For a vector $v=\left(v_{j}\right)_{j=1}^{J} \in \mathbf{R}^{J}$ and $f=\left(f_{j}\right)_{j=1}^{J} \in$ $\oplus^{J} L^{2}\left(\mathbf{R}^{d}\right)$, we define

$$
C(v, f):=\sum_{j=1}^{J} v_{j}^{2}\|f\|_{L^{2}\left(\mathbf{R}^{d}\right)}^{2} .
$$

We set

$$
a:=\left(a_{j}\right)_{j=1}^{J}, \quad b:=\left(b_{j}\right)_{j=1}^{J}
$$


Proposition 3.6 For all $\nu \in\left(0, \nu_{0}\right)$ and $j=1, \cdots, J$,

$$
R_{j}(\nu) \leq \frac{a_{j}^{2}\left[E_{0}(\nu)-\mathcal{E}_{0}(A)+\alpha^{2} C(b, \lambda / \sqrt{\omega})\right]}{1-\alpha^{2} C(a, \lambda / \sqrt{\omega})}+b_{j}^{2}
$$

In partiular,

$$
\sup _{\nu \in\left(0, \nu_{0}\right)} R_{j}(\nu) \leq \frac{a_{j}^{2}\left[E_{0}\left(\nu_{0}\right)-\mathcal{E}_{0}(A)+\alpha^{2} C(b, \lambda / \sqrt{\omega})\right]}{1-\alpha^{2} C(a, \lambda / \sqrt{\omega})}+b_{j}^{2}<\infty .
$$

Remark 3.2 (i) Condition (2.4) implies that

$$
\alpha^{2} C(a, \lambda / \sqrt{\omega})<1
$$

see (2.18). (ii) If $H$ has a ground state $\Psi_{0}$ and we define $R_{j}(0):=\left\|B_{j} \otimes I \Psi_{0}\right\|^{2}$, then (3.30) with $\nu=0$ holds, see the proof below.

Proof. By (2.6), we have

$$
R_{j}(\nu) \leq a_{j}^{2}\left\|\widetilde{A}^{1 / 2} \otimes I \Psi_{0}(\nu)\right\|^{2}+b_{j}^{2} .
$$

On the other hand, we have

$$
\begin{aligned}
\left\|\widetilde{A}^{1 / 2} \otimes I \Psi_{0}(\nu)\right\|^{2} & =\left\langle\left(H(\nu)-I \otimes d \Gamma\left(\hat{\omega}_{\nu}\right)-\alpha H_{I}\right)\right\rangle_{\nu}-\mathcal{E}_{0}(A) \\
& =E_{0}(\nu)-\mathcal{E}_{0}(A)+\langle A \otimes I\rangle_{\nu}-\nu n(\nu)-\langle H\rangle_{\nu} \\
& \leq E_{0}(\nu)-\mathcal{E}_{0}(A)+\langle A \otimes I\rangle_{\nu}-\langle H\rangle_{\nu} .
\end{aligned}
$$

It follows from the proof of Proposition 2.3 that

$$
\langle H\rangle_{\nu} \geq q_{L \otimes I}\left(\Psi_{0}(\nu), \Psi_{0}(\nu)\right) .
$$

Hence

$$
\left\|\widetilde{A}^{1 / 2} \otimes I \Psi_{0}(\nu)\right\|^{2} \leq E_{0}(\nu)-\mathcal{E}_{0}(A)+\alpha^{2} R
$$

where $R:=\sum_{j=1}^{J}\left\|\lambda_{j}\right\|_{-1}^{2} R_{j}(\nu)$. Thus

$$
R_{j}(\nu) \leq a_{j}^{2}\left(E_{0}(\nu)-\mathcal{E}_{0}(A)+\alpha^{2} R\right)+b_{j}^{2} .
$$

Multiplying $\left\|\lambda_{j}\right\|_{-1}^{2}$ to the both sides and taking the summation in $j$, we obtain

$$
R \leq \frac{C(a, \lambda / \sqrt{\omega})\left(E_{0}(\nu)-\mathcal{E}_{0}(A)\right)+C(b, \lambda / \sqrt{\omega})}{1-\alpha^{2} C(a, \lambda / \sqrt{\omega})} .
$$

Putting this into (3.33), we obtain (3.30).

Estimate (3.31) follows from (3.30) and the monotone nondecreasing property of $E_{0}(\nu)$ in $m$ [Proposition 3.2(i)]. 
Corollary 3.7 Suppose that $\lambda_{j} / \omega \in L^{2}\left(\mathbf{R}^{d}\right), j=1, \cdots, J$. Then

$$
\bar{n} \leq \alpha^{2}\left(\sum_{j=1}^{J}\left\|\lambda_{j} / \omega\right\|_{L^{2}\left(\mathbf{R}^{d}\right)} \sqrt{\frac{a_{j}^{2}\left[E_{0}(0)-\mathcal{E}_{0}(A)+\alpha^{2} C(b, \lambda / \sqrt{\omega})\right]}{1-\alpha^{2} C(a, \lambda / \sqrt{\omega})}+b_{j}^{2}}\right)^{2} .
$$

Proof. This follows from Corollary 3.5, Proposition 3.6 and the easily proven fact that $\left\|\lambda_{j} / \omega_{\nu}\right\|_{L^{2}\left(\mathbf{R}^{d}\right)} \rightarrow\left\|\lambda_{j} / \omega\right\|_{L^{2}\left(\mathbf{R}^{d}\right)}$ as $\nu \rightarrow 0$

\subsection{Infrared divergence}

In concluding this section we give a sufficient condition for $\underline{n}=\bar{n}=+\infty$ (infrared divergence in the mean boson number).

Theorem 3.8 Let $\left\{\lambda_{1}, \cdots, \lambda_{J}, \omega\right\}$ obey the infrared singularity condition (1.7). Suppose that there exists a function $g$ on the set $S_{K}:=\left\{k \in \mathbf{R}^{d} \mid \omega(k) \leq K\right\}$ (K>0 is a constant) such that, for a.e. $k \in S_{K}, \lambda_{j}(k)=g(k), j=1, \cdots, J$. Moreover, suppose that

$$
\liminf _{\nu \rightarrow 0}\left|\left\langle\sum_{j=1}^{J} B_{j} \otimes I\right\rangle_{\nu}\right|>0
$$

Then

$$
\lim _{\nu \rightarrow 0} n(\nu)=+\infty
$$

Proof. Using the identity

$$
\left(H(\nu) \Psi_{0}(\nu), I \otimes a(f) \Psi_{0}(\nu)\right)-\left(I \otimes a(f)^{*} \Psi_{0}(\nu), H(\nu) \Psi_{0}(\nu)\right)=0, \quad f \in L^{2}\left(\mathbf{R}^{d}\right),
$$

and commutation relations, we can show that, for all $f \in L^{2}\left(\mathbf{R}^{d}\right)$,

$$
\langle I \otimes a(f)\rangle_{\nu}=-\sum_{j=1}^{J} \alpha\left(f, \frac{\lambda_{j}}{\omega_{\nu}}\right)_{L^{2}\left(\mathbf{R}^{d}\right)}\left\langle B_{j} \otimes I\right\rangle_{\nu} .
$$

Hence

$$
\begin{aligned}
\left|\sum_{j=1}^{J} \alpha\left(f, \frac{\lambda_{j}}{\omega_{\nu}}\right)_{L^{2}\left(\mathbf{R}^{d}\right)}\left\langle B_{j} \otimes I\right\rangle_{\nu}\right| & \leq\left\|I \otimes a(f) \Psi_{0}(\nu)\right\| \\
& \leq\|f\|_{L^{2}\left(\mathbf{R}^{d}\right)}\left\|I \otimes N_{\mathrm{b}}^{1 / 2} \Psi_{0}(\nu)\right\| \\
& =\|f\|_{L^{2}\left(\mathbf{R}^{d}\right)} \sqrt{n(\nu)} .
\end{aligned}
$$

Taking $f=g \chi_{S_{K}} / \omega_{\nu}$ and putting

$$
C_{\nu}:=\int_{S_{K}} \frac{|g(k)|^{2}}{\omega_{\nu}(k)^{2}} d k
$$


we obtain

$$
|\alpha| \sqrt{C_{\nu}}\left|\left\langle\sum_{j=1}^{J} B_{j} \otimes I\right\rangle_{\nu}\right| \leq \sqrt{n(\nu)} .
$$

By (1.7), $C_{\nu} \rightarrow+\infty$ as $\nu \rightarrow 0$. Thus (3.36) follows.

\section{Existence of Ground States of $H$}

Throughout this section, we assume (H.1)-(H.3). Our aim here is to give a sufficient condition, in terms of quantities defined from the regularized theory, for existence of ground states of $H$.

\subsection{Existence theorem}

Definition 4.1 We denote by $\mathcal{G}_{\Psi_{0}}$ the set of all non-zero vectors $\Psi \in \mathcal{F}$ such that, for a sequence $\left\{\nu_{j}\right\}_{j=1}^{\infty} \subset\left(0, \nu_{0}\right)$ satisfying $\nu_{j} \downarrow 0$ as $j \rightarrow \infty$,

$$
\mathrm{w}-\lim _{j \rightarrow \infty} \Psi_{0}\left(\nu_{j}\right)=\Psi
$$

where $\mathrm{w}$ - lim means weak limit.

Lemma 4.2 Suppose that $\mathcal{G}_{\Psi_{0}} \neq \emptyset$. Then:

(i) Every vector in $\mathcal{G}_{\Psi_{0}}$ is a ground state of $H$.

(ii) If $\underline{n}<\infty$, then $\mathcal{G}_{\Psi_{0}} \subset D\left(I \otimes N_{\mathrm{b}}^{1 / 2}\right)$ and, for all $\Psi \in \mathcal{G}_{\Psi_{0}}$

$$
\left\|I \otimes N_{\mathrm{b}}^{1 / 2} \Psi\right\|^{2} \leq \underline{n} .
$$

Proof. (i) Since we have Proposition 3.1(i) and (3.12), we can apply [15, Lemma 4.9] to obtain the desired result.

(ii) Let $\Psi \in \mathcal{G}_{\Psi_{0}}$ such that (4.1) holds and $\mathcal{F}_{n}:=\mathcal{H} \otimes\left(\otimes_{\mathrm{s}}^{n} L^{2}\left(\mathbf{R}^{d}\right)\right)$ so that $\mathcal{F}=\oplus_{n=0}^{\infty} \mathcal{F}_{n}$. Then, for all $N \in \mathbf{N}$, we have

$$
\sum_{n=1}^{N} n\left\|\Psi_{0}\left(\nu_{j}\right)^{(n)}\right\|_{\mathcal{F}_{n}}^{2} \leq n\left(\nu_{j}\right)
$$

Let $\left\{\Phi_{\ell}^{(n)}\right\}_{\ell=1}^{\infty}$ be a complete orthonormal system of $\mathcal{F}_{n}$. By the Parseval equality, we have for all $M \in \mathbf{N}$

$$
\sum_{n=1}^{N} \sum_{\ell=1}^{M} n\left|\left(\Psi_{0}\left(\nu_{j}\right)^{(n)}, \Phi_{\ell}^{(n)}\right)_{\mathcal{F}_{n}}\right|^{2} \leq n\left(\nu_{j}\right)
$$


By (4.1), $\lim _{j \rightarrow \infty}\left(\Psi_{0}\left(\nu_{j}\right)^{(n)}, \Phi_{\ell}^{(n)}\right)_{\mathcal{F}_{n}}=\left(\Psi^{(n)}, \Phi_{\ell}^{(n)}\right)_{\mathcal{F}_{n}}$. Hence, taking $j \rightarrow \infty \operatorname{in}(4.3)$ first and then $M \rightarrow \infty$, we obtain

$$
\sum_{n=1}^{N} \sum_{\ell=1}^{\infty} n\left|\left(\Psi^{(n)}, \Phi_{\ell}^{(n)}\right)_{\mathcal{F}_{n}}\right|^{2} \leq \underline{n}
$$

i.e., for all $N \in \mathbf{N}$,

$$
\sum_{n=1}^{N} n\left\|\Psi^{(n)}\right\|^{2} \leq \underline{n}
$$

which implies that $\Psi \in D\left(I \otimes N_{\mathrm{b}}^{1 / 2}\right)$ and (4.2) holds.

Let

$$
H_{I}^{(-)}:=\sum_{j=1}^{J} B_{j}^{*} \otimes a\left(\lambda_{j}\right)
$$

and

$$
W(\nu):=\left\|H_{I}^{(-)} \Psi_{0}(\nu)\right\|^{2}=\sum_{j, \ell=1}^{J}\left(B_{j}^{*} \otimes a\left(\lambda_{j}\right) \Psi_{0}(\nu), B_{\ell}^{*} \otimes a\left(\lambda_{\ell}\right) \Psi_{0}(\nu)\right) .
$$

We define

$$
\bar{W}:=\limsup _{\nu \downarrow 0} W(\nu) .
$$

Let $\sigma_{\text {ess }}(A)$ be the essential spectrum of $A$ and set

$$
\Sigma:=\inf \sigma_{\mathrm{ess}}(A),
$$

provided that $\sigma_{\text {ess }}(A) \neq \emptyset$. We assume the following:

(H.4) $\Sigma>\mathcal{E}_{0}(A)$.

This assumption is only for the case where $\sigma_{\text {ess }}(A) \neq \emptyset$. Under (H.4), $\mathcal{E}_{0}(A)$ belongs to the discrete spectrum of $A$, so that it is an eigenvalue of $A$ with finite multiplicity.

Theorem 4.3 Assume (H.1)-(H.3).

(i) Let $\sigma_{\text {ess }}(A) \neq \emptyset$ and (H.4) be satisfied. Suppose that

$$
\bar{n}+\frac{\alpha^{2} \bar{W}}{\left(\Sigma-E_{0}(0)\right)^{2}}<1 .
$$

Then $\mathcal{G}_{\Psi_{0}} \neq \emptyset, \mathcal{G}_{\Psi_{0}} \subset D\left(I \otimes N_{\mathrm{b}}^{1 / 2}\right)$ and every vector in $\mathcal{G}_{\Psi_{0}}$ is a ground state of $H$.

(ii) Let $\sigma_{\mathrm{ess}}(A)=\emptyset$. Suppose that

$$
\bar{n}<1 \text {. }
$$

Then $\mathcal{G}_{\Psi_{0}} \neq \emptyset, \mathcal{G}_{\Psi_{0}} \subset D\left(I \otimes N_{\mathrm{b}}^{1 / 2}\right)$ and every vector $\Psi$ in $\mathcal{G}_{\Psi_{0}}$ is a ground state of $H$. 
Remark 4.1 Since

$$
n(\nu)=\frac{E_{0}(\nu)-\langle H\rangle_{\nu}}{\nu}, \quad \nu>0
$$

(cf. (A.14) in Appendix A), we have

$$
\bar{n}=\limsup _{\nu \rightarrow 0} \frac{E_{0}(\nu)-\langle H\rangle_{\nu}}{\nu} .
$$

Hence, conditions (4.8) and (4.9) can be rewritten in terms of energy expectation values (together with the correlation function $W(\nu)$ in the case $\left.\sigma_{\mathrm{ess}}(A) \neq \emptyset\right)$.

The following corollary gives sufficient conditions, in terms of $E_{0}(0)$ and the right differential $E_{0}^{\prime}(0+)$ of $E_{0}(\nu)$ at $\nu=0$, for $H$ to have a ground state.

Corollary 4.4 Assume (H.1)-(H.3).

(i) Let $\sigma_{\text {ess }}(A) \neq \emptyset$ and (H.4) be satisfied. Suppose that $E_{0}(\nu)$ has the right differential $E_{0}^{\prime}(0+)$ at $\nu=0$ as a function of $\nu$ and

$$
E_{0}^{\prime}(0+)+\frac{\alpha^{2} \bar{W}}{\left(\Sigma-E_{0}(0)\right)^{2}}<1
$$

Then $\mathcal{G}_{\Psi_{0}} \neq \emptyset, \mathcal{G}_{\Psi_{0}} \subset D\left(I \otimes N_{\mathrm{b}}^{1 / 2}\right)$ and every vector in $\mathcal{G}_{\Psi_{0}}$ is a ground state of $H$.

(ii) Let $\sigma_{\mathrm{ess}}(A)=\emptyset$. Suppose that $E_{0}(\nu)$ has the right differential $E_{0}^{\prime}(0+)$ at $\nu=0$ as a function of $\nu$ and

$$
E_{0}^{\prime}(0+)<1 .
$$

Then $\mathcal{G}_{\Psi_{0}} \neq \emptyset, \mathcal{G}_{\Psi_{0}} \subset D\left(I \otimes N_{\mathrm{b}}^{1 / 2}\right)$ and every vector in $\mathcal{G}_{\Psi_{0}}$ is a ground state of $H$.

To prove Theorem 4.3, we need a lemma. Let $P_{A}$ be the spectral measure of $A$ and, for $r>\mathcal{E}_{0}(A), Q_{r}$ be the orthogonal projection from $\mathcal{H}$ onto the range of $P_{A}\left(\left[\mathcal{E}_{0}(A), r\right)\right.$ ), where in the case $\sigma_{\text {ess }}(A) \neq \emptyset$, we impose the condition $r<\Sigma$ too. Then $Q_{r}$ is finite rank. We define

$$
Q_{r}^{\perp}:=I-Q_{r}
$$

We denote by $P_{\Omega}$ the orthogonal projection from $\mathcal{F}_{\mathrm{b}}$ onto the one-dimensional subspace $\{c \Omega \mid c \in \mathbf{C}\}$ generated by the Fock vacuum $\Omega$ in $\mathcal{F}_{\mathrm{b}}$.

Lemma 4.5 For all $\nu \in\left(0, \nu_{0}\right)$,

$$
\left\langle Q_{r}^{\perp} \otimes P_{\Omega}\right\rangle_{\nu} \leq \frac{\alpha^{2} W(\nu)}{\left(r-E_{0}(\nu)\right)^{2}} .
$$

Remark 4.2 We have by (3.10) $r>\mathcal{E}_{0}(A) \geq E_{0}(\nu)$, so that $r>E_{0}(\nu)$. 
Proof. Since $d \Gamma\left(\widehat{\omega}_{\nu}\right) \Omega=0, a(f) \Omega=0\left(f \in L^{2}\left(\mathbf{R}^{d}\right)\right)$, and $P_{\Omega} \psi=(\Omega, \psi)_{\mathcal{F}_{\mathrm{b}}} \Omega\left(\psi \in \mathcal{F}_{\mathrm{b}}\right)$, we have $I \otimes P_{\Omega} H(\nu)=A \otimes P_{\Omega}+\alpha I \otimes P_{\Omega} H_{I}^{(-)}$on $D(H(\nu))$, so that

$$
Q_{r}^{\perp} \otimes P_{\Omega} H(\nu)=Q_{r}^{\perp} A \otimes P_{\Omega}+\alpha Q_{r}^{\perp} \otimes P_{\Omega} H_{I}^{(-)}
$$

on $D(H(\nu))$. Noting that $Q_{r}^{\perp} A Q_{r}^{\perp} \geq r$, we can show, in the same way as in the proof of [15, Lemma 4.7], that

$$
\left\langle Q_{r}^{\perp} \otimes P_{\Omega}\right\rangle_{\nu} \leq \frac{|\alpha|}{r-E_{0}(\nu)}\left\|Q_{r}^{\perp} \otimes P_{\Omega} \Psi_{0}(\nu)\right\|\left\|H_{I}^{(-)} \Psi_{0}(\nu)\right\|,
$$

which implies (4.14).

\section{Proof of Theorem 4.3}

Let $\Psi \in \mathcal{G}_{\Psi_{0}}$. Then, for a sequence $\left\{\nu_{j}\right\}_{j=1}^{\infty} \subset\left(0, \nu_{0}\right)$ satisfying $\nu_{j} \downarrow 0$ as $j \rightarrow \infty,(4.1)$ holds.

By [15, Lemma 4.6],

$$
Q_{r} \otimes P_{\Omega} \geq I-I \otimes N_{\mathrm{b}}-Q_{r}^{\perp} \otimes P_{\Omega}
$$

which implies that

$$
\left\langle Q_{r} \otimes P_{\Omega}\right\rangle_{\nu} \geq 1-n(\nu)-\left\langle Q_{r}^{\perp} \otimes P_{\Omega}\right\rangle_{\nu}
$$

Hence, by Lemma 4.5, we obtain

$$
\left\langle Q_{r} \otimes P_{\Omega}\right\rangle_{\nu} \geq 1-n(\nu)-\frac{\alpha^{2} W(\nu)}{\left(r-E_{0}(\nu)\right)^{2}} .
$$

We first consider the case where $\operatorname{dim} \mathcal{H}=\infty$. Since $Q_{r} \otimes P_{\Omega}$ is a finite-rank orthogonal projection, it follows that

$$
\lim _{j \rightarrow \infty}\left\langle Q_{r} \otimes P_{\Omega}\right\rangle_{\nu_{j}}=\left(\Psi, Q_{r} \otimes P_{\Omega} \Psi\right)
$$

Hence

$$
\left(\Psi, Q_{r} \otimes P_{\Omega} \Psi\right) \geq 1-\bar{n}-\frac{\alpha^{2} \bar{W}}{\left(r-E_{0}(0)\right)^{2}},
$$

where we have used that

$$
\lim _{\nu \rightarrow \infty} E_{0}(\nu)=E_{0}(0)
$$

[see Proposition 3.2(iii)].

If $\sigma_{\text {ess }}(A) \neq \emptyset$, then

$$
\liminf _{r \rightarrow \Sigma}\left(\Psi, Q_{r} \otimes P_{\Omega} \Psi\right) \geq 1-\bar{n}-\frac{\alpha^{2} \bar{W}}{\left(\Sigma-E_{0}(0)\right)^{2}} .
$$


Under condition (4.8), the right hand side is positive. Hence $\Psi \neq 0$. Now we can apply [15, Lemma 4.9] to conclude that $\Psi$ is a ground state of $H$.

If $\sigma_{\text {ess }}(A)=\emptyset$ or $\operatorname{dim} \mathcal{H}<\infty$, then $Q(r) \rightarrow I$ as $r \rightarrow \infty$. Hence, taking $r \rightarrow \infty$ in (4.15), we have

$$
\left(\Psi, I \otimes P_{\Omega} \Psi\right) \geq 1-\bar{n} .
$$

Thus, under condition (4.9), $\Psi \neq 0$. Hence $\mathcal{G}_{\Psi_{0}} \neq \emptyset$ and Lemma 4.2 completes the proof.

\section{Proof of Corollary 4.4}

We need only combine Theorem 4.3 and Proposition 3.3(iv).

\section{$4.2 \quad$ Upper bound for $W(\nu)$}

Let

$$
U_{\nu}^{(a, b)}:=\left\{\left(\varepsilon, \varepsilon^{\prime}\right) \in(0, \infty) \times(0, \infty)|| \alpha \mid F_{\varepsilon, \varepsilon^{\prime}}^{(a, b)}\left(\lambda, \omega_{\nu}\right)<1\right\}, \quad \nu \geq 0,
$$

and, for $\eta>0$ and $\left(\varepsilon, \varepsilon^{\prime}\right) \in U_{\nu}^{(a, b)}$,

$$
\begin{aligned}
h_{\eta, \varepsilon, \varepsilon^{\prime}}(\nu):= & \frac{\left(M_{a}\left(\lambda / \sqrt{\omega_{\nu}}\right)+\sqrt{2} J \eta\right)\left(\left|E_{0}(\nu)\right|+\left|\mathcal{E}_{0}(A)\right|+|\alpha| G_{\varepsilon, \varepsilon^{\prime}}^{(a, b)}\left(\lambda, \omega_{\nu}\right)\right)}{\sqrt{2}\left(1-|\alpha| F_{\varepsilon, \varepsilon^{\prime}}^{(a, b)}\left(\lambda, \omega_{\nu}\right)\right)} \\
& +\frac{C\left(b, \lambda / \sqrt{\omega_{\nu}}\right)}{4 \eta} .
\end{aligned}
$$

Let

$$
h(\nu):=\inf _{\eta>0,\left(\varepsilon, \varepsilon^{\prime}\right) \in U_{\nu}^{(a, b)}} h_{\eta, \varepsilon, \varepsilon^{\prime}}(\nu) .
$$

Proposition 4.6 We have

$$
W(\nu) \leq h(\nu)^{2} .
$$

In particular,

$$
\bar{W} \leq h(0)
$$

Proof. Let $\Psi \in D\left(H_{0}\right)$. Then, by (2.6),

$$
\left\|B_{j}^{*} \otimes a\left(\lambda_{j}\right) \Psi\right\| \leq a_{j}\left\|\widetilde{A}^{1 / 2} \otimes a\left(\lambda_{j}\right) \Psi\right\|+b_{j}\left\|I \otimes a\left(\lambda_{j}\right) \Psi\right\| .
$$

Using the well known basic estimates

$$
\begin{aligned}
\|a(f) \psi\| \leq & \|f\|_{-1}\left\|d \Gamma(\omega)^{1 / 2} \psi\right\|, \\
\left\|a(f)^{*} \psi\right\|^{2} \leq & \|f\|_{-1}^{2}\left\|d \Gamma(\omega)^{1 / 2} \psi\right\|^{2}+\|f\|^{2}\|\psi\|^{2}, \\
& \quad \psi \in D\left(d \Gamma(\omega)^{1 / 2}\right), f \in \mathcal{M}_{0} \cap \mathcal{M}_{-1},
\end{aligned}
$$


we have

$$
\begin{aligned}
\left\|\widetilde{A}^{1 / 2} \otimes a\left(\lambda_{j}\right) \Psi\right\| & \leq\left\|\lambda_{j}\right\|_{-1}\left\|\left(\widetilde{A}^{1 / 2} \otimes I\right)\left(I \otimes d \Gamma(\omega)^{1 / 2}\right) \Psi\right\| \\
& \leq \frac{1}{\sqrt{2}}\left\|\lambda_{j}\right\|_{-1}\left\|\widetilde{H}_{0} \Psi\right\|
\end{aligned}
$$

and

$$
\begin{aligned}
b_{j}\left\|I \otimes a\left(\lambda_{j}\right) \Psi\right\| & \leq b_{j}\left\|\lambda_{j}\right\|_{-1}\left\|\widetilde{H}_{0}^{1 / 2} \Psi\right\| \\
& \leq b_{j}\left\|\lambda_{j}\right\|_{-1}\|\Psi\|^{1 / 2}\left\|\widetilde{H}_{0} \Psi\right\|^{1 / 2} \\
& \leq \eta\left\|\widetilde{H}_{0} \Psi\right\|+\frac{b_{j}^{2}}{4 \eta}\left\|\lambda_{j}\right\|_{-1}^{2}\|\Psi\|,
\end{aligned}
$$

where $\eta$ is an abitrary positive constant. Hence

$$
\left\|B_{j}^{*} \otimes a\left(\lambda_{j}\right) \Psi\right\| \leq\left(\frac{a_{j}}{\sqrt{2}}\left\|\lambda_{j}\right\|_{-1}+\eta\right)\left\|\widetilde{H}_{0} \Psi\right\|+\frac{b_{j}^{2}}{4 \eta}\left\|\lambda_{j}\right\|_{-1}^{2}\|\Psi\|,
$$

which gives

$$
\left\|H_{I}^{(-)} \Psi\right\| \leq\left(\frac{M_{a}(\lambda / \sqrt{\omega})}{\sqrt{2}}+J \eta\right)\left\|\widetilde{H}_{0} \Psi\right\|+\frac{C(b, \lambda / \sqrt{\omega})}{4 \eta}\|\Psi\| .
$$

On the other hand, by (2.16), we have

$$
\begin{aligned}
\left\|\widetilde{H}_{0} \Psi\right\| & =\left\|\left(H-\alpha H_{I}-\mathcal{E}_{0}(A)\right) \Psi\right\| \\
& \leq\|H \Psi\|+|\alpha|\left\|H_{I} \Psi\right\|+\left|\mathcal{E}_{0}(A)\right|\|\Psi\| \\
& \leq\|H \Psi\|+|\alpha| F_{\varepsilon, \varepsilon^{\prime}}^{(a, b)}(\lambda, \omega)\left\|\widetilde{H}_{0} \Psi\right\|+|\alpha| G_{\varepsilon, \varepsilon^{\prime}}^{(a, b)}(\lambda, \omega)\|\Psi\|+\left|\mathcal{E}_{0}(A)\right|\|\Psi\| .
\end{aligned}
$$

Hence

$$
\left\|\widetilde{H}_{0} \Psi\right\| \leq \frac{1}{1-|\alpha| F_{\varepsilon, \varepsilon^{\prime}}^{(a, b)}(\lambda, \omega)}\left(\|H \Psi\|+|\alpha| G_{\varepsilon, \varepsilon^{\prime}}^{(a, b)}(\lambda, \omega)\|\Psi\|+\left|\mathcal{E}_{0}(A)\right|\|\Psi\|\right),
$$

where $\left(\varepsilon, \varepsilon^{\prime}\right) \in U_{0}^{(a, b)}$. In particular, taking $\left(\varepsilon, \varepsilon^{\prime}\right) \in U_{\nu}^{(a, b)}$, we have

$$
\left\|\widetilde{H}_{0} \Psi_{0}(\nu)\right\| \leq \frac{1}{1-|\alpha| F_{\varepsilon, \varepsilon^{\prime}}^{(a, b)}\left(\lambda, \omega_{\nu}\right)}\left(\left|E_{0}(\nu)\right|+|\alpha| G_{\varepsilon, \varepsilon^{\prime}}^{(a, b)}\left(\lambda, \omega_{\nu}\right)+\left|\mathcal{E}_{0}(A)\right|\right) .
$$

Hence we obtain

$$
W(\nu)^{1 / 2}=\left\|H_{I}^{(-)} \Psi_{0}(\nu)\right\| \leq h_{\eta, \varepsilon, \varepsilon^{\prime}}(\nu)
$$

Thus the desired result follows.

Remark 4.3 Estimate (4.20) holds also in the case $\nu=0$ if $H$ has a ground state $\Psi_{0}(0)$ and we set $W(0):=\left\|H_{I}^{(-)} \Psi_{0}(0)\right\|^{2}$. 


\section{Existence of Ground States in the Regularized The- ory}

In this section we establish an existence theorem of ground states of the $H$ with $\mu>0$. Since ess.inf $\operatorname{liR}_{k \in \mathbf{R}^{d}} \omega_{\nu}(k) \geq \nu$ with $\nu>0$, the case of $H(\nu)$ is included in the case we are going to treat.

\subsection{Preliminary results}

We denote by $P_{A}$ the spectral measure of $A$ as before. In the case where $\sigma_{\text {ess }}(A) \neq \emptyset$, assuming (H.4) and fixing a parameter $s$ with condition

$$
0<s<\Sigma-\mathcal{E}_{0}(A)
$$

we set

$$
P:=P_{A}\left(\left[\mathcal{E}_{0}(A), \Sigma-s\right]\right),
$$

while, in the case $\sigma_{\text {ess }}(A)=\emptyset$, we set $P=I$. Then $P$ is an orthogonal projection. We set

$$
Q:=I-P,
$$

so that

$$
Q P=P Q=0 .
$$

Let

$$
\hat{P}:=P \otimes I, \quad \hat{Q}:=Q \otimes I,
$$

acting on $\mathcal{F}$. The Hilbert space $\mathcal{F}$ is decomposed as

$$
\mathcal{F}=\mathcal{F}_{P} \oplus \mathcal{F}_{Q}
$$

with

$$
\begin{aligned}
\mathcal{F}_{P} & :=\operatorname{Ran}(\hat{P})=\operatorname{Ran}(P) \otimes \mathcal{F}_{\mathrm{b}} \\
\mathcal{F}_{Q} & :=\operatorname{Ran}(\hat{Q})=\operatorname{Ran}(Q) \otimes \mathcal{F}_{\mathrm{b}}
\end{aligned}
$$

We first consider the operator

$$
H_{1}:=H_{0}+\alpha H_{I, P},
$$

where

$$
H_{I, P}:=\sum_{j=1}^{J}\left(\left(P B_{j} P+Q B_{j} Q\right) \otimes a\left(\lambda_{j}\right)^{*}+\left(P B_{j}^{*} P+Q B_{j}^{*} Q\right) \otimes a\left(\lambda_{j}\right)\right)
$$


Lemma 5.1 Assume (H.1) and (H.2). Then $D\left(H_{0}\right) \subset D\left(H_{I, P}\right), H_{1}$ is self-adjoint on $D\left(H_{1}\right)=D\left(H_{0}\right)$ and bounded from below. Moreover, $H_{1}$ is essentially self-adjoint on every core of $H_{0}$.

Proof. Putting

$$
\widetilde{B}_{j}:=P B_{j} P+Q B_{j} Q, \quad \widetilde{B}_{j}^{\dagger}:=P B_{j}^{*} P+Q B_{j}^{*} Q,
$$

we have

$$
H_{I, P}=\sum_{j=1}^{J}\left(\widetilde{B}_{j} \otimes a\left(\lambda_{j}\right)^{*}+\widetilde{B}_{j}^{\dagger} \otimes a\left(\lambda_{j}\right)\right) .
$$

It is easy to see that, for all $u \in D\left(\widetilde{A}^{1 / 2}\right)$,

$$
\left\|\frac{\left(\widetilde{B}_{j}^{\dagger} \pm \widetilde{B}_{j}\right)}{\sqrt{2}} u\right\|^{2} \leq a_{j, \pm}^{2}\left\|\widetilde{A}^{1 / 2} u\right\|^{2}+b_{j, \pm}^{2}\|u\|^{2}, \quad j=1, \cdots, J .
$$

Hence, in the same way as in the proof of Proposition 2.1, we can show that

$$
\left\|H_{I, P} \Psi\right\| \leq F_{\varepsilon, \varepsilon^{\prime}}^{(a, b)}(\lambda, \omega)\left\|\widetilde{H}_{0} \Psi\right\|+G_{\varepsilon, \varepsilon^{\prime}}^{(a, b)}(\lambda, \omega)\|\Psi\|, \Psi \in D\left(H_{0}\right) .
$$

Thus the Kato-Rellich theorem gives the desired result.

We want to establish the existence of a ground state of $H_{1}$. For this purpose, we assume the following.

(H.5) The function $\omega(k)$ is continuous with

$$
\lim _{|k| \rightarrow \infty} \omega(k)=\infty
$$

and there exist constant $\gamma>0$ and $C>0$ such that

$$
\left|\omega(k)-\omega\left(k^{\prime}\right)\right| \leq C\left|k-k^{\prime}\right|^{\gamma}\left(1+\omega(k)+\omega\left(k^{\prime}\right)\right), \quad k, k^{\prime} \in \mathbf{R}^{d} .
$$

For $f=\left(f_{j}\right)_{j=1}^{J} \in \oplus^{J} L^{2}\left(\mathbf{R}^{d}\right)$, we define

$$
S_{A}(f):=\Sigma-\alpha^{2} C(a, f)\left[\Sigma-\mathcal{E}_{0}(A)\right]-\alpha^{2} C(b, f),
$$

where $C(\cdot, f)$ is defined by (3.28).

Theorem 5.2 Consider the case $\sigma_{\mathrm{ess}}(A) \neq \emptyset$ and assume (H.1), (H.2), (H.4), (H.5) and $\mu>0$. Suppose that

$$
S_{A}(\lambda / \sqrt{\omega})>\mathcal{E}_{0}\left(H_{1}\right)
$$

so that

$$
M_{0}:=\min \left\{\mu, S_{A}(\lambda / \sqrt{\omega})-\mathcal{E}_{0}\left(H_{1}\right)\right\}>0 .
$$

Then $H_{1}$ has purely discrete spectrum in the interval $\left[\mathcal{E}_{0}\left(H_{1}\right), \mathcal{E}_{0}\left(H_{1}\right)+M_{0}\right)$. 
Remark 5.1 In the same way as in the case of $H$, we can show that

$$
\mathcal{E}_{0}\left(H_{1}\right) \leq \mathcal{E}_{0}(A)
$$

cf. (2.27). Hence, if

$$
\Sigma-\mathcal{E}_{0}(A)>\frac{\alpha^{2} C(b, \lambda / \sqrt{\omega})}{1-\alpha^{2} C(a, \lambda / \sqrt{\omega})},
$$

then (5.11) holds [note (3.32)].

Remark 5.2 In the case $\sigma_{\mathrm{ess}}(A)=\emptyset$, under the assumptions (H.1), (H.2) and (H.5), the conclusion of Theorem 5.2 holds with $M_{0}=\mu$. This is just [15, Theorem 1.2]. In the cited theorem, the continuity of $\lambda_{j}$ is also assumed. However this is not needed in fact, because $H_{1 P}$ is approximated by $H_{1 P}$ with $\lambda_{j}$ continuous in norm-resolvent sense.

To prove this theorem, we need lemmas: Let

$$
H_{1 P}:=P H P\left|\mathcal{F}_{P}, \quad H_{1 Q}:=Q H Q\right| \mathcal{F}_{Q} .
$$

Then we have

$$
H_{1}=H_{1 P} \oplus H_{1 Q}
$$

We define

$$
S_{A, s}(f):=\Sigma-s-\alpha^{2} C(a, f)\left(\Sigma-s-\mathcal{E}_{0}(A)\right)-\alpha^{2} C(b, f) .
$$

Lemma 5.3 Assume (H.1) and (H.2). Suppose that $\sigma_{\mathrm{ess}}(A) \neq \emptyset$. Then

$$
H_{1 Q} \geq S_{A, s}(\lambda / \sqrt{\omega}) .
$$

Proof. In the same way as in the proof of Proposition 2.3, we have

$$
\begin{aligned}
H_{1 Q} \geq & (Q A Q) \otimes I-\sum_{j=1}^{J} \alpha^{2}\left\|\frac{\lambda_{j}}{\sqrt{\omega}}\right\|_{L^{2}\left(\mathbf{R}^{d}\right)}^{2}\left(Q B_{j}^{*} B_{j} Q\right) \otimes I \\
\geq & (Q A Q) \otimes I-\alpha^{2} C(a, \lambda / \sqrt{\omega})(Q \widetilde{A} Q) \otimes I \\
& \quad-\alpha^{2} C(b, \lambda / \sqrt{\omega}) \\
\geq & \left(1-\alpha^{2} C(a, \lambda / \sqrt{\omega})\right) Q(A \otimes I) Q \\
& +\alpha^{2} C(a, \lambda / \sqrt{\omega}) \mathcal{E}_{0}(A)-\alpha^{2} C(b, \lambda / \sqrt{\omega}) \\
\geq & \left(1-\alpha^{2} C(a, \lambda / \sqrt{\omega})\right)(\Sigma-s)+\alpha^{2} C(a, \lambda / \sqrt{\omega}) \mathcal{E}_{0}(A) \\
& \quad-\alpha^{2} C(b, \lambda / \sqrt{\omega})
\end{aligned}
$$

in the sense of quadratic form, where we have used (3.32). Hence (5.17) follows.

Lemma 5.4 Under the same assumption as in Theorem 5.2, $H_{1 P}$ has purely discrete spectrum in the interval $\left[\mathcal{E}_{0}\left(H_{1 P}\right), \mathcal{E}_{0}\left(H_{1 P}\right)+\mu\right)$.

Proof. Note that $\operatorname{dim} \operatorname{Ran}(P)<\infty$. Hecne $A \mid \operatorname{Ran}(P)$ has purely discrete spectrum consisting of a finite number of eigenvalues. Hence, in quite the same way as in the proof of [15, Theorem 1.2], we can prove the present lemma (cf. Remark 5.2). 


\section{Proof of Theorem 5.2}

By (5.15), we have $\sigma\left(H_{1}\right)=\sigma\left(H_{1 P}\right) \cup \sigma\left(H_{1 Q}\right)$. Hence Lemmas 5.3 and 5.4 imply that $\mathcal{E}_{0}\left(H_{1 P}\right)=\mathcal{E}_{0}\left(H_{1}\right)$ and that $H_{1}$ has purely discrete spectrum in

$$
\left[\mathcal{E}_{0}\left(H_{1}\right), \mathcal{E}_{0}\left(H_{1}\right)+\min \left\{\mu, S_{A, s}(\lambda / \sqrt{\omega})-\mathcal{E}_{0}\left(H_{1}\right)\right\}\right) .
$$

Since $s$ is arbitrary with (5.1), we obtain the desired result.

\subsection{Main result}

As for existence of ground states of $H$, we need the following additional assumption.

(H.6) There exist constants $c_{j, \pm}$ and $d_{j, \pm}(j=1, \cdots, J)$ such that, for all $u \in D\left(\widetilde{A}^{1 / 2}\right)$,

$$
\begin{aligned}
& \left\|\frac{Q\left(B_{j}^{*} \pm B_{j}\right) P}{\sqrt{2}} u\right\|^{2}+\left\|\frac{P\left(B_{j}^{*} \pm B_{j}\right) Q}{\sqrt{2}} u\right\|^{2} \leq c_{j, \pm}^{2}\left\|\widetilde{A}^{1 / 2} u\right\|^{2}+d_{j, \pm}^{2}\|u\|^{2}, \\
& j=1, \cdots, J,
\end{aligned}
$$

and

$$
|\alpha| \sum_{j=1}^{J}\left(c_{j,+}+c_{j,-}\right)\left\|\lambda_{j}\right\|_{-1}<1 .
$$

Remark 5.3 This assumption is non-trivial only in the case $\sigma_{\mathrm{ess}}(A) \neq \emptyset$, because, if $\sigma_{\mathrm{ess}}(A)=\emptyset$, then $Q=0$ so that (5.18) and (5.19) trivially hold with $c_{j, \pm}=d_{j, \pm}=0$.

Remark 5.4 The constants $c_{j, \pm}$ and $d_{j, \pm}$ may be small. For example, If each $B_{j}$ is bounded, then we can take

$$
c_{j, \pm}=0, \quad d_{j, \pm}=\frac{1}{2}\left(\left\|\left[Q, B_{j}^{*} \pm B_{j}\right] P\right\|^{2}+\left\|\left[P, B_{j}^{*} \pm B_{j}\right] Q\right\|^{2}\right),
$$

where $[X, Y]:=X Y-Y X$. The quantities $\left\|\left[Q, B_{j}^{*} \pm B_{j}\right] P\right\|^{2}+\left\|\left[P, B_{j}^{*} \pm B_{j}\right] Q\right\|^{2}$ may be small (an extreme case is given by the one where $\left[Q, B_{j}^{*} \pm B_{j}\right] P=0$ and $\left[P, B_{j}^{*} \pm B_{j}\right] Q=0$ ).

Let

$$
v(c):=\left(c_{j,+}+c_{j,-}\right)_{j=1}^{J}, \quad v(d):=\left(d_{j,+}+d_{j,-}\right)_{j=1}^{J} \in \mathbf{R}^{J}
$$

and, for $f=\left(f_{j}\right)_{j=1}^{J} \in \oplus^{J} L^{2}\left(\mathbf{R}^{d}\right)$,

$$
\begin{aligned}
C_{\varepsilon, \varepsilon^{\prime}}(f, \omega) & :=\frac{|\alpha| F_{\varepsilon, \varepsilon^{\prime}}^{(c, d)}(f, \omega)}{1-|\alpha| F_{\varepsilon, \varepsilon^{\prime}}^{(a, b)}(f, \omega)} \\
D_{\varepsilon, \varepsilon^{\prime}}(f, \omega) & :=C_{\varepsilon, \varepsilon^{\prime}}(f, \omega)\left(|\alpha| G_{\varepsilon, \varepsilon^{\prime}}^{(a, b)}(f, \omega)+\left|\mathcal{E}_{0}(A)\right|\right)+|\alpha| G_{\varepsilon, \varepsilon^{\prime}}^{(c, d)}(f, \omega) .
\end{aligned}
$$


where $\left(\varepsilon, \varepsilon^{\prime}\right) \in U_{0}^{(a, b)}$.

Let

$$
\mathcal{E}_{1}\left(H_{1}\right):=\inf \sigma\left(H_{1}\right) \cap\left(\mathcal{E}_{0}\left(H_{1}\right), \infty\right)
$$

and

$$
r_{0}:=\frac{\mathcal{E}_{1}\left(H_{1}\right)+\mathcal{E}_{0}\left(H_{1}\right)}{2}-\mathcal{E}_{0}(H) .
$$

It follows from (5.15) that

$$
\mathcal{E}_{0}\left(H_{1}\right) \geq \mathcal{E}_{0}(H)
$$

Hence

$$
r_{0}>\frac{\mathcal{E}_{1}\left(H_{1}\right)-\mathcal{E}_{0}\left(H_{1}\right)}{2}>0
$$

Theorem 5.5 Let $\sigma_{\mathrm{ess}}(A) \neq \emptyset$. Assume (H.1), (H.2), (H.4), (H.5), (H.6) and $\mu>0$. Suppose that

$$
\begin{aligned}
\inf _{\left(\varepsilon, \varepsilon^{\prime}\right) \in U_{0}^{(a, b)} \cap U_{0}^{(c, d)}} \quad\left\{C_{\varepsilon, \varepsilon^{\prime}}(\lambda, \omega)+\frac{2\left[D_{\varepsilon, \varepsilon^{\prime}}(\lambda, \omega)+C_{\varepsilon, \varepsilon^{\prime}}(\lambda, \omega)\left(r_{0}+\left|\mathcal{E}_{0}(H)\right|\right)\right]}{\mathcal{E}_{1}\left(H_{1}\right)-\mathcal{E}_{0}\left(H_{1}\right)}\right\} \\
<\frac{\mathcal{E}_{1}\left(H_{1}\right)-\mathcal{E}_{0}\left(H_{1}\right)}{\mathcal{E}_{1}\left(H_{1}\right)-\mathcal{E}_{0}\left(H_{1}\right)+2} .
\end{aligned}
$$

Then $H$ has purely discrete spectrum in $\left[\mathcal{E}_{0}(H), \mathcal{E}_{0}(H)+r_{0}\right)$. In particular, $H$ has a ground state.

Proof. We can write as

$$
H=H_{1}+H_{2}
$$

with

$$
H_{2}=\alpha \sum_{j=1}^{J}\left(\left(P B_{j} Q+Q B_{j} P\right) \otimes a\left(\lambda_{j}\right)^{*}+\left(P B_{j}^{*} Q+Q B_{j}^{*} P\right) \otimes a\left(\lambda_{j}\right)\right) .
$$

In the same way as in Proposition 2.1, using (H.6), we can show that, for all $\Psi \in D\left(H_{0}\right)$,

$$
\left\|H_{2} \Psi\right\| \leq|\alpha| F_{\varepsilon, \varepsilon^{\prime}}^{(c, d)}(\lambda, \omega)\left\|\widetilde{H}_{0} \Psi\right\|+|\alpha| G_{\varepsilon, \varepsilon^{\prime}}^{(c, d)}(\lambda, \omega)\|\Psi\| .
$$

On the other hand, we have [cf. (4.24)]

$$
\left\|\widetilde{H}_{0} \Psi\right\| \leq \frac{1}{1-|\alpha| F_{\varepsilon, \varepsilon^{\prime}}^{(a, b)}(\lambda, \omega)}\left(\left\|H_{1} \Psi\right\|+|\alpha| G_{\varepsilon, \varepsilon^{\prime}}^{(a, b)}(\lambda, \omega)\|\Psi\|+\left|\mathcal{E}_{0}(A)\right|\|\Psi\|\right)
$$

for all $\Psi \in D\left(H_{0}\right)$. Hence

$$
\left\|H_{2} \Psi\right\| \leq C_{\varepsilon, \varepsilon^{\prime}}(\lambda, \omega)\left\|H_{1} \Psi\right\|+D_{\varepsilon, \varepsilon^{\prime}}(\lambda, \omega)\|\Psi\| .
$$

Thus applying Corollary B.2 in Appendix B, we obtain the desired result. 


\subsection{Existence of a ground state in the case $\mu=0$ with infrared regularity condition}

Theorem 5.6 Consider the case where $\sigma_{\mathrm{ess}}(A) \neq \emptyset$ and $\mu=0$, but, $\lambda_{j} / \omega \in L^{2}\left(\mathbf{R}^{d}\right), j=$

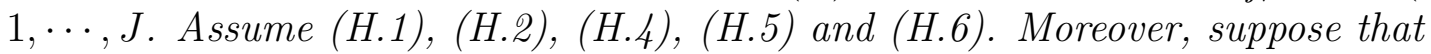

$$
\begin{aligned}
\alpha^{2}\left(\sum_{j=1}^{J}\left\|\lambda_{j} / \omega\right\|_{L^{2}\left(\mathbf{R}^{d}\right)}\right. & \left.\sqrt{\frac{a_{j}^{2}\left[E_{0}(0)-\mathcal{E}_{0}(A)+\alpha^{2} C(b, \lambda / \sqrt{\omega})\right]}{1-\alpha^{2} C(a, \lambda / \sqrt{\omega})}+b_{j}^{2}}\right)^{2} \\
& +\frac{\alpha^{2} h(0)}{\left(\Sigma-E_{0}(0)\right)^{2}}<1 .
\end{aligned}
$$

Then $H$ has a ground state.

Proof. This follows from Theorem 4.3(i), Proposition 4.6 and Corollary 3.7.

\section{Examples}

In this section we discuss some simple examples in each of which one can explicitly see a correspondence between existence (or absence) of ground states and the right differentiability of the ground-state energy $E_{0}(\nu)$ of the regularized model at $\nu=0$ (cf. Corollary 4.4).

\subsection{The van Hove Model}

Let $\mathcal{H}=\mathbf{C}, J=1, A=0, B_{1}=1 / \sqrt{2}$, and $\lambda_{j}=\lambda$. Then the Hamiltonian $H$ defined by (1.4) takes the form

$$
H_{\mathrm{VH}}=d \Gamma(\widehat{\omega})+\alpha \phi(\lambda)
$$

with condition

$$
\lambda, \quad \frac{\lambda}{\sqrt{\omega}} \in L^{2}\left(\mathbf{R}^{\nu}\right) .
$$

This gives the so-called van Hove model (e.g., [25, Chapter 1, §e] and references therein). We denote by $E_{0, \mathrm{VH}}$ the ground-state energy of $H_{\mathrm{vH}}$.

Theorem 6.1 (i)

$$
E_{0, \mathrm{VH}}=-\frac{1}{2} \alpha^{2}\left\|\frac{\lambda}{\sqrt{\omega}}\right\|_{L^{2}\left(\mathbf{R}^{d}\right)}^{2} .
$$

(ii) Let $\mu>0$. Then $H_{\mathrm{VH}}$ has a unique ground state (up to constant multiples) given by

$$
\Omega_{\mathrm{VH}}:=e^{i \alpha \phi(i \lambda / \omega)} \Omega .
$$


Moreover,

$$
\left(\Omega_{\mathrm{VH}}, N_{\mathrm{b}} \Omega_{\mathrm{VH}}\right)=\frac{1}{2} \alpha^{2}\left\|\frac{\lambda}{\omega}\right\|_{L^{2}\left(\mathbf{R}^{d}\right)}^{2} .
$$

(iii) Let $\mu=0$. Then $H_{\mathrm{VH}}$ has a ground state if and only if $\lambda / \omega \in L^{2}\left(\mathbf{R}^{d}\right)$. In that case the ground state is unique up to constant multiples and given by $\Omega_{\mathrm{VH}}$ and (6.5) holds.

Proof. (i) It is well known (or easy to see) that (6.3) holds if $\mu>0$ ([25, Chapter 1 , $\S \mathrm{e}]$ and references therein). Then, applying Proposition 3.2(iii), we obtain (6.3) also in the case $\mu=0$.

(ii) This also is well known ([25, Chapter $1, \S \mathrm{e}]$ and references therein).

(iii) See [16, Remark 3.6].

Theorem 6.1(iii) can be rephrased as follows:

Corollary 6.2 Consider the case $\mu=0$. Then $H_{\mathrm{VH}}$ has no ground states if and only if the infrared singularity condition (1.7) with $\lambda_{j}=\lambda$ is satisfied.

We denote by $E_{0, \mathrm{VH}}(\nu)$ the ground-state energy of the regularized Hamiltonian

$$
H_{\mathrm{VH}}(\nu):=d \Gamma\left(\widehat{\omega_{\nu}}\right)+\alpha \phi(\lambda), \quad \nu \geq 0 .
$$

Applying Theorem 6.1(i) with $\omega$ replaced by $\omega_{\nu}$, we have

$$
E_{0, \mathrm{VH}}(\nu)=-\frac{1}{2} \alpha^{2}\left\|\frac{\lambda}{\sqrt{\omega_{\nu}}}\right\|_{L^{2}\left(\mathbf{R}^{d}\right)}^{2}, \quad \nu \geq 0 .
$$

Using this expression, one can easily prove the following fact:

Proposition 6.3 (i) The function $E_{0, \mathrm{VH}}(\nu)$ is differentiable on $(0, \infty)$ with

$$
E_{0, \mathrm{VH}}^{\prime}(\nu)=\frac{1}{2} \alpha^{2}\left\|\frac{\lambda}{\omega_{\nu}}\right\|_{L^{2}\left(\mathbf{R}^{d}\right)}^{2}, \quad \nu>0 .
$$

(ii) The function $E_{0, \mathrm{VH}}(\nu)$ is right differentiable at $\nu=0$ if and only if $\lambda / \omega \in L^{2}\left(\mathbf{R}^{d}\right)$. In that case,

$$
E_{0, \mathrm{VH}}^{\prime}(0+)=\lim _{\nu \rightarrow 0} E_{0, \mathrm{VH}}^{\prime}(\nu)=\frac{1}{2} \alpha^{2}\left\|\frac{\lambda}{\omega}\right\|_{L^{2}\left(\mathbf{R}^{d}\right)}^{2} .
$$


By Theorem 6.1(iii) and Proposition 6.3(ii), we see that, in the case $\mu=0, H_{\mathrm{VH}}$ has a ground state if and only if $E_{0, \mathrm{VH}}(\nu)$ is right differentiable at $\nu=0$. Thus, in this example, the existence of ground states of $H_{\mathrm{VH}}$ without low-energy cutoff can be completely characterized in terms of the right differentiability of $E_{0, \mathrm{VH}}(\nu)$ at $\nu=0$.

Let $\Omega_{\mathrm{VH}}(\nu)$ be the vector $\Omega_{\mathrm{VH}}$ with $\omega$ replaced by $\omega_{\nu}$, i.e., a ground state of $H_{\mathrm{VH}}(\nu)$ and

$$
n_{\mathrm{VH}}(\nu):=\left(\Omega_{\mathrm{VH}}(\nu), N_{\mathrm{b}} \Omega_{\mathrm{VH}}(\nu)\right), \quad \nu>0 .
$$

Then, by (6.5), we have

$$
n_{\mathrm{VH}}(\nu)=\frac{1}{2} \alpha^{2}\left\|\frac{\lambda}{\omega_{\nu}}\right\|_{L^{2}\left(\mathbf{R}^{d}\right)}^{2} .
$$

Consider the case $\mu=0$. Then

$$
\bar{n}_{\mathrm{VH}}:=\lim _{\nu \rightarrow 0} n_{\mathrm{vH}}(\nu)
$$

exists and finite if and only if $\lambda / \omega \in L^{2}\left(\mathbf{R}^{d}\right)$. Thus, in this example, the existence of ground states of $H_{\mathrm{VH}}$ without low-energy cutoff can be completely characterized also in terms of the existence of $\bar{n}_{\mathrm{VH}}$. Note that, if $\lambda / \omega \notin L^{2}\left(\mathbf{R}^{d}\right)$ (infrared singularity condition), then $\bar{n}_{\mathrm{VH}}=+\infty$.

\subsection{A quantum harmonic oscillator coupled to a Bose field}

We consider the case where

$$
\mathcal{H}=\mathcal{F}_{\mathrm{b}}(\mathbf{C})=\oplus_{n=0}^{\infty} \mathbf{C},
$$

the Boson Fock space over the one-dimensional Hilbert space $\mathbf{C}$, and $J=1$. We denote by $b(z)$ the annihilation operator on $\mathcal{F}_{\mathbf{b}}(\mathbf{C})$ with test vector $z \in \mathbf{C}$ so that, for all $z, w \in \mathbf{C}$,

$$
\left[b(z), b(w)^{*}\right]=z^{*} w, \quad[b(z), b(w)]=0=\left[b(z)^{*}, b(w)^{*}\right],
$$

on the subspace of finite particle vectors in $\mathcal{F}_{\mathrm{b}}(\mathbf{C})$. We set

$$
b:=b(1) .
$$

Let $\mu_{0} \in \mathbf{R}$ and take $A$ and $B_{1}$ as

$$
A=\mu_{0} b^{*} b, \quad B_{1}=b .
$$

Then the Hamiltonian $H$ defined by (1.4) takes the form

$$
H_{\mathrm{RWA}}=\mu_{0} b^{*} b \otimes I+I \otimes d \Gamma(\widehat{\omega})+\alpha\left(b \otimes a(\lambda)^{*}+b^{*} \otimes a(\lambda)\right)
$$

acting in the Hilbert space

$$
\mathcal{F}_{\text {RWA }}:=\mathcal{F}_{\mathrm{b}}(\mathbf{C}) \otimes \mathcal{F}_{\mathrm{b}},
$$

where we assume (6.2). This model is called the rotating-wave-approximation (RWA) oscillator. A detailed analysis for this model with $\mu_{0}>0$ is given in [7](cf. also [19] for a 
recent development). Here we do not restrict ourselves to the case $\mu_{0}>0$, although the model with $\mu_{0} \leq 0$ does not belong to the class of the GSB model. Also we present a method different from that in [7].

We first note that $\mathcal{F}_{\text {RWA }}$ is identified in a natural way with the Boson Fock space $\mathcal{F}_{\mathrm{b}}(\mathcal{W})$ over the Hilbert space

$$
\mathcal{W}:=\mathbf{C} \oplus L^{2}\left(\mathbf{R}^{d}\right)
$$

i.e.,

$$
\mathcal{F}_{\text {RWA }}=\mathcal{F}_{\mathrm{b}}(\mathcal{W}) .
$$

The unitary correspondence which gives this identification is as follows:

$$
\begin{aligned}
& e_{0} \otimes \Omega \longleftrightarrow \Omega_{\mathcal{W}}, \\
& b \otimes I \longleftrightarrow a(\langle 1,0\rangle) \\
& I \otimes a(f) \longleftrightarrow a(\langle 0, f\rangle), \quad f \in L^{2}\left(\mathbf{R}^{d}\right),
\end{aligned}
$$

where $e_{0}\left(\operatorname{resp} . \Omega_{\mathcal{W}}\right)$ is the Fock vacuum in $\mathcal{F}_{\mathrm{b}}(\mathbf{C})\left(\operatorname{resp} . \mathcal{F}_{\mathrm{b}}(\mathcal{W})\right)$ and $a(\langle z, f\rangle)(\langle z, f\rangle \in \mathcal{W})$ is the annihilation operator on $\mathcal{F}_{\mathrm{b}}(\mathcal{W})$ ) with test vector $\langle z, f\rangle$. In what follows we use this identification freely. We also use a simpler notation $a(z, f)$ for $a(\langle z, f\rangle)$.

Let

$$
h_{0}:=\left(\begin{array}{cc}
\mu_{0} & 0 \\
0 & \hat{\omega}
\end{array}\right), \quad h_{1}:=\left(\begin{array}{cc}
0 & (\lambda, \cdot)_{L^{2}\left(\mathbf{R}^{d}\right)} \\
\lambda & 0
\end{array}\right)
$$

acting on $\mathcal{W}$ and define

$$
h:=h_{0}+\alpha h_{1} .
$$

It is obvious that $h_{0}$ is self-adjoint with $D\left(h_{0}\right)=\mathbf{C} \oplus D(\hat{\omega})$ and bounded from below with

$$
h_{0} \geq \min \left\{\mu_{0}, \mu\right\} .
$$

It is easy to see that $h_{1}$ is a bounded self-adjoint operator with

$$
\left\|h_{1}\right\| \leq\|\lambda\|_{L^{2}\left(\mathbf{R}^{d}\right)} .
$$

Hence $h$ is self-adjoint with $D(h)=D\left(h_{0}\right)$ and bounded from below.

Remark 6.1 The operator $h$ is the Hamiltonian of a model of Friedrichs's type [26] (cf. also [37] and references therein for recent developments).

The long-time behavior of the matrix element $\left(\psi_{0}, e^{-i t h} \psi_{0}\right)$ with $\psi_{0}:=(1,0)$ as $t \rightarrow \infty$ is analyzed in [38] in the case $\omega(k)=|k|$.

We introduce a subspace

$$
\mathcal{F}_{\text {fin }}(h):=\mathcal{L}\left\{a\left(z_{1}, f_{1}\right)^{*} \cdots a\left(z_{n}, f_{n}\right)^{*} \Omega_{\mathcal{W}} \mid n \geq 0, z_{j} \in \mathbf{C}, f_{j} \in D(\hat{\omega})\right\} .
$$

Under the identification (6.9), we have

$$
\mathcal{F}_{\text {fin }}(h)=\mathcal{L}\left\{b^{* m} e_{0} \otimes a\left(f_{1}\right)^{*} \cdots a\left(f_{n}\right)^{*} \Omega \mid m, n \geq 0, f_{j} \in D(\hat{\omega})\right\} .
$$

We denote by $d \Gamma(h)$ the second quantization of $h$. A key fact is the following. 
Lemma 6.4 Under the identification (6.9),

$$
\bar{H}_{\mathrm{RWA}}=d \Gamma(h) .
$$

In particular, $H_{\mathrm{RWA}}$ is essentially self-adjoint on $\mathcal{F}_{\text {fin }}(h)$.

Proof. One first shows by direct computation that, for all $\Psi \in \mathcal{F}_{\text {fin }}(h), H_{\mathrm{RWA}} \Psi=$ $d \Gamma(h) \Psi$. Since $\mathcal{F}_{\text {fin }}(h)$ is a core of $d \Gamma(h)$, (6.14) follows as well as the essential selfadjointness of $H_{\text {RWA }}$ on $\mathcal{F}_{\text {fin }}(h)$.

Remark 6.2 In Lemma 6.4, we do not assume that $\mu_{0}>0, \omega \lambda \in L^{2}\left(\mathbf{R}^{d}\right)$ (in fact, we do not need the condition $\lambda / \sqrt{\omega} \in L^{2}\left(\mathbf{R}^{d}\right)$ either as is seen). This improves a result on the essential self-adjointness of $H_{\mathrm{RWA}}$ established in [7, Proposition 2.1].

By Lemma 6.4, the spectral analysis of $H_{\mathrm{RWA}}$ is reduced to that of $h$. To analyze spectral properties of $h$, we introduce a function

$$
D(z):=-z+\mu_{0}+\alpha^{2} \int_{\mathbf{R}^{d}} \frac{|\lambda(k)|^{2}}{z-\omega(k)} d k
$$

defined for all $z \in \mathbf{C}$ such that $|\lambda(k)|^{2} /|z-\omega(k)|$ is Lebesgue integrable on $\mathbf{R}^{d}$. In particular, $D(z)$ is defined in the cut plane

$$
\mathbf{C}_{\mu}:=\mathbf{C} \backslash[\mu, \infty)
$$

and analytic there. It is easy to see that $D(x)$ is monotone decreasing in $x<\mu$. Hence the limit

$$
d_{\mu}:=\lim _{x \uparrow \mu} D(x)=-\mu+\mu_{0}-\lim _{\varepsilon \downarrow 0} \alpha^{2} \int_{\mathbf{R}^{d}} \frac{|\lambda(k)|^{2}}{\omega(k)-\mu+\varepsilon} d k
$$

exists, being allowed to be $-\infty$.

Lemma 6.5 (i) If $d_{\mu} \geq 0$, then $D(z)$ has no zeros in $\mathbf{C}_{\mu}$.

(ii) If $d_{\mu}<0$, then $D(z)$ has a unique simple zero $x_{0} \in(-\infty, \mu)$. In particular, if $\mu>0$ and $\mu_{0} \geq \alpha^{2}\|\lambda / \sqrt{\omega}\|_{L^{2}\left(\mathbf{R}^{d}\right)}^{2}$, then $0 \leq x_{0}<\mu$; if $\mu_{0}<\alpha^{2}\|\lambda / \sqrt{\omega}\|_{L^{2}\left(\mathbf{R}^{d}\right)}^{2}$, then $x_{0}<0$.

Proof. An easy exercise (cf. [7, Lemma 3.1]).

For a self-adjoint operator $T$, we denote by $\sigma_{\text {ess }}(T)$ its essential spectrum.

Lemma 6.6 Suppose that $\omega$ is continuous on $\mathbf{R}^{d}$. Then

$$
\left\{\omega(k) \mid k \in \mathbf{R}^{d}\right\} \subset \sigma_{\mathrm{ess}}(h) .
$$


Proof. Let $a \in\left\{\omega(k) \mid k \in \mathbf{R}^{d}\right\}$. Then there is a point $k_{a} \in \mathbf{R}^{d}$ such that $\omega\left(k_{a}\right)=a$. Let $\xi \in C_{0}^{\infty}(\mathbf{R})$ such that $\xi(k)=0$ for $|k| \geq 1$ and $\int_{\mathbf{R}^{d}} \xi(k)^{2} d k=1$ and, for $n \geq 1$, define $f_{n}$ by

$$
f_{n}(k):=n^{d / 2} \xi\left(n\left(k-k_{a}\right)\right) .
$$

Then $f_{n} \in D(\hat{\omega})$ with $\left\|f_{n}\right\|_{L^{2}\left(\mathbf{R}^{d}\right)}=1$. Let $\psi_{n}:=\left\langle 0, f_{n}\right\rangle \in \mathcal{W}$. Then $\left\|\psi_{n}\right\|=1$ and

$$
(h-a) \psi_{n}=\left\langle\alpha\left(\lambda, f_{n}\right)_{L^{2}\left(\mathbf{R}^{d}\right)},(\hat{\omega}-a) f_{n}\right\rangle .
$$

Hence

$$
\left\|(h-a) \psi_{n}\right\|^{2}=\alpha^{2}\left|\left(\lambda, f_{n}\right)_{L^{2}\left(\mathbf{R}^{d}\right)}\right|^{2}+\left\|(\hat{\omega}-a) f_{n}\right\|_{L^{2}\left(\mathbf{R}^{d}\right)}^{2} .
$$

It is easy to see that, for all $g \in L^{2}\left(\mathbf{R}^{d}\right)$,

$$
\lim _{n \rightarrow \infty}\left(g, f_{n}\right)_{L^{2}\left(\mathbf{R}^{d}\right)}=0
$$

(first prove this for $g$ continuous, then use a limiting argument). In particular, $\lim _{n \rightarrow \infty}$ $\left|\left(\lambda, f_{n}\right)_{L^{2}\left(\mathbf{R}^{d}\right)}\right|^{2}=0$. Moreover we can show that $\lim _{n \rightarrow \infty}\left\|(\hat{\omega}-a) f_{n}\right\|_{L^{2}\left(\mathbf{R}^{d}\right)}^{2}=0$. Hence $\lim _{n \rightarrow \infty}\left\|(h-a) \psi_{n}\right\|^{2}=0$. (6.19) implies that $\mathrm{w}-\lim _{n \rightarrow \infty} \psi_{n}=0$. Thus, by a basic criterion on essential spectrum ([2, Lemma 5.19]), we conclude that $a \in \sigma_{\text {ess }}(h)$. Thus (6.18) holds.

Lemma 6.7 (i) If $d_{\mu} \geq 0$, then $(-\infty, \mu) \subset \varrho(h)(\varrho(h)$ denotes the resolvent set of $h)$.

(ii) If $d_{\mu}<0,(-\infty, \mu) \backslash\left\{x_{0}\right\} \subset \varrho(h)$ and $x_{0}$ is a simple eigenvalue of $h$.

Proof. Let $x \in(-\infty, \mu)$ and $D(x) \neq 0$. Then we want to show that $x \in \varrho(h)$. For this purpose, let $\langle z, f\rangle \in \mathcal{W}$. Then we define

$$
y:=\frac{z+\alpha\left(\lambda, \frac{f}{\omega-x}\right)_{L^{2}\left(\mathbf{R}^{d}\right)}}{D(x)}, \quad g:=\frac{f-\alpha y \lambda}{\omega-x} .
$$

These quantities are well-defined with $g \in D(\hat{\omega})$, so that $\langle y, g\rangle \in D(h)$. Moreover we see that $(h-x)\langle y, g\rangle=\langle z, f\rangle$. Hence $h-x$ is surjective. The equation $(h-x)\langle z, f\rangle=0$ is equivalent to

$$
\left(\mu_{0}-x\right) z+\alpha(\lambda, f)_{L^{2}\left(\mathbf{R}^{d}\right)}=0, \quad(\omega-x) f+z \alpha \lambda=0 .
$$

Putting the second equation into the first, we obtain $z D(x)=0$. Hence $z=0$. Then, by the second equation, $(\omega-x) f=0$, which implies that $f=0$. Hence $h-x$ is injective. Thus $x \in \varrho(h)$. ¿From this fact and Lemma 6.5 we obtain part (i) and the assertion on $\varrho(h)$ in part (ii). To show that $x_{0}$ is a simple eigenvalue of $h$ in the case $d_{\mu}<0$, we consider the eigenvector equation: $h\langle z, f\rangle=E\langle z, f\rangle$ with $\langle z, f\rangle \in D(h)$ and $E \in(-\infty, \mu)$. Then (6.20) with $x=E$ holds. Hence $z D(E)=0$. Suppose that $E \neq x_{0}$. Then $D(E) \neq 0$ by Lemma 6.5(ii). Hence $z=0$ and $f=0$ as is shown above. This means $E$ cannot be an 
eigenvalue of $h$. Hence, if $h$ has an eigenvalue $E \in(-\infty, \mu)$, then $E=x_{0}$. Moreover, the vector

$$
\phi_{0}:=\left\langle 1,-\alpha \lambda /\left(\omega-x_{0}\right)\right\rangle
$$

is an eigenvector of $h$ : $\phi_{0} \in D(h)$ and $h \phi_{0}=x_{0} \phi_{0}$. Thus $x_{0}$ is an eigenvalue of $h$. The simplicity of $x_{0}$ easily follows from the eigenvalue equation of $h$.

Remark 6.3 In the case $d_{\mu}<0$, we have

$$
\left\|\phi_{0}\right\|^{2}=-D^{\prime}\left(x_{0}\right)
$$

With these preliminaries we can prove the following theorem.

Theorem 6.8 Assume the following:

(RWA) $\omega$ is continuous on $\mathbf{R}^{d}$ and $\omega(k) \rightarrow \infty$ as $|k| \rightarrow \infty$.

(i) Let $d_{\mu} \geq 0$. Then

$$
\sigma(h)=[\mu, \infty)
$$

(ii) Let $d_{\mu}<0$. Then

$$
\sigma(h)=\left\{x_{0}\right\} \cup[\mu, \infty),
$$

where $x_{0}$ is a simple eigenvalue of $h$ with eigenvector $\phi_{0}$.

Proof. By (RWA),

$$
\left\{\omega(k) \mid k \in \mathbf{R}^{d}\right\}=[\mu, \infty) .
$$

Hence, by Lemma 6.6

$$
[\mu, \infty) \subset \sigma_{\text {ess }}(h) .
$$

(i) In this case we have by Lemma 6.7(i) $\sigma(h) \subset[\mu, \infty)$. By this fact and (6.25) we obtain (6.23).

(ii) In this case we have by Lemma 6.7(ii) $\sigma(h) \subset\left\{x_{0}\right\} \cup[\mu, \infty)$. By this fact and (6.25) we obtain (6.24).

Remark 6.4 In both cases (i) and (ii), h may have eigenvalues in $[\mu, \infty)$. For example, consider the case $\lambda(k)=0$ for $|k| \geq \kappa$ with a constant $\kappa>0$. Let $\mu(\kappa):=\sup _{|k| \leq \kappa} \omega(k)<$ $\infty$ and suppose that $\lim _{x \downarrow \mu(\kappa)} \int_{|k| \leq \kappa} d k|\lambda(k)|^{2} /(x-\omega(k))=+\infty$. Then $D(z)$ has a unique simple zero $y_{0}$ in $(\mu(\kappa), \infty)$, which is an eigenvalue of $h$ (cf. also [19]).

If we assume, in addition to (RWA), that, for all $x \in[\mu, \infty),|\lambda(k)|^{2} /|x-\omega(k)|$ is not Lebesgue integrable, then $h$ has no eigenvalues in $[\mu, \infty)$. Hence, in this case, Theorem $6.8(i)$ shows that, under condition $d_{\mu} \geq 0$, the eignevalue $\mu_{0}$ of the unperturbed operator $h_{0}$ is unstable under the perturbation $\alpha h_{1}$, namely, it disappears under the perturbation $\alpha h_{1}$. 
For a linear operator $T$, we denote by $\sigma_{\mathrm{p}}(T)$ the point spectrum of $T$.

Theorem 6.9 Let the same assumption as in Theorem 6.8 be satisfied.

(i) Let $d_{\mu} \geq 0$. Then

$$
\sigma\left(\bar{H}_{\mathrm{RWA}}\right)=\{0\} \cup[\mu, \infty)
$$

where 0 is a simple eigenvalue with eigenvector $e_{0} \otimes \Omega$. In particular, $\bar{H}_{\mathrm{RWA}}$ is nonnegative with ground-state energy $\mathcal{E}_{0}\left(\bar{H}_{\mathrm{RWA}}\right)=0$.

(ii) Let $d_{\mu}<0, \mu>0$ and $\mu_{0} \geq \alpha^{2}\|\lambda / \sqrt{\omega}\|_{L^{2}\left(\mathbf{R}^{d}\right)}^{2}$. Then,

$$
\begin{aligned}
& \sigma\left(\bar{H}_{\mathrm{RWA}}\right)=\{0\} \cup\left\{n x_{0}\right\}_{n=1}^{\infty} \cup[\mu, \infty), \\
& \{0\} \cup\left\{n x_{0}\right\}_{n=1}^{\infty} \subset \sigma_{\mathrm{p}}\left(\bar{H}_{\mathrm{RWA}}\right),
\end{aligned}
$$

with $x_{0} \geq 0$, where 0 is a simple eigenvalue with eigenvector $e_{0} \otimes \Omega$ and $n x_{0}$ is a simple eigenvalue of $\bar{H}_{\mathrm{RWA}}$ with eigenvector $a\left(\phi_{0}\right)^{* n} \Omega_{\mathcal{W}}$. In particular, $\bar{H}_{\mathrm{RWA}}$ is nonnegative with ground-state energy $\mathcal{E}_{0}\left(\bar{H}_{\mathrm{RWA}}\right)=0$.

(iii) Let $\mu_{0}<\alpha^{2}\|\lambda / \sqrt{\omega}\|_{L^{2}\left(\mathbf{R}^{d}\right)}^{2}\left(\right.$ hence $\left.d_{\mu}<0\right)$. Then,

$$
\sigma\left(\bar{H}_{\mathrm{RWA}}\right)=\mathbf{R}, \quad\{0\} \cup\left\{n x_{0}\right\}_{n=1}^{\infty}=(-\infty, \mu) \cap \sigma_{\mathrm{p}}\left(\bar{H}_{\mathrm{RWA}}\right),
$$

with $x_{0}<0$, where 0 is a simple eigenvalue with eigenvector $e_{0} \otimes \Omega$ and $n x_{0}$ is a simple eigenvalue of of $\bar{H}_{\mathrm{RWA}}$ with eigenvector $a\left(\phi_{0}\right)^{* n} \Omega_{\mathcal{W}}$. In particular, $\bar{H}_{\mathrm{RWA}}$ is neither bounded from below nor above.

Proof. By (6.14) and the spectral property of the second quantization operator, we have

$$
\sigma\left(\bar{H}_{\mathrm{RWA}}\right)=\{0\} \bigcup\left(\cup_{n=1}^{\infty}\left\{\sum_{j=1}^{n} s_{j} \mid s_{j} \in \sigma(h), j=1, \cdots, n\right\}\right),
$$

where 0 is an eigenvalue with eigenvector $e_{0} \otimes \Omega$. By this formula and Theorem 6.8 we obtain the desired result.

Remark 6.5 Consider the case $d_{\mu} \geq 0$. Then $\mu_{0} \geq \mu$ and hence the positive eigenvalues $\left\{n \mu_{0}\right\}_{n=1}^{\infty}$ of the unperturbed Hamiltonian $H_{\mathrm{RWA}, 0}:=\mu_{0} b^{*} b \otimes I+I \otimes d \Gamma(\hat{\omega})$ are embedded in its essential spectrum $\sigma_{\mathrm{ess}}\left(H_{\mathrm{RWA}, 0}\right)=[\mu, \infty)$. We have $\sigma(h)=[\mu, \infty)$ (Theorem $6.8(i))$. Suppose in addition that $\sigma_{\mathrm{p}}(h)=\emptyset$. Then we have $\sigma_{\mathrm{p}}\left(\bar{H}_{\mathrm{RWA}}\right) \cap(0, \infty)=\emptyset$, since $\sigma_{\mathrm{p}}(d \Gamma(h)) \cap(0, \infty)=\emptyset$. Hence, in this case, all the embedded eigenvalues $\left\{n \mu_{0}\right\}_{n=1}^{\infty}$ of $H_{\mathrm{RWA}, 0}$ disappear under the perturbation $\alpha\left(b^{*} \otimes a(\lambda)+b \otimes a(\lambda)^{*}\right)$.

We want to remark that, in the cases (ii) and (iii) of Theorem 6.9, the Hilbert space $\mathcal{F}_{\text {RWA }}$ has a special orthogonal decomposition as is shown below. Suppose that the assumption of (ii) or (iii) of Theorem 6.9 is satisfied and $\mathcal{X}_{0}$ be the one-dimensional subspace generated by the vector $\phi_{0}$

$$
\mathcal{X}_{0}:=\left\{z \phi_{0} \mid z \in \mathbf{C}\right\}
$$


Let

$$
\mathcal{W}^{\prime}=\mathcal{X}_{0} \oplus \mathcal{X}_{0}^{\perp}
$$

For all $\langle z, f\rangle \in \mathcal{W}$, we have the orthogonal decomposition

$$
\langle z, f\rangle=C_{z, f} \phi_{0}+\psi_{z, f}
$$

with respect to the vector $\phi_{0}$, where $C_{z, f}:=\left(\phi_{0},\langle z, f\rangle\right)_{\mathcal{W}} /\left\|\phi_{0}\right\|_{\mathcal{W}}^{2}$ and $\psi_{z, f}$ is a vector such that $\left(\phi_{0}, \psi_{z, f}\right)_{\mathcal{W}}=0$. Hence the oprator $u: \mathcal{W} \rightarrow \mathcal{W}^{\prime}$ defined by

$$
u\langle z, f\rangle:=\left\langle C_{z, f} \phi_{0}, \psi_{z, f}\right\rangle .
$$

is unitary. This gives an identification of $\mathcal{W}$ with $\mathcal{W}^{\prime}$. Hence $\mathcal{F}_{\mathrm{b}}(\mathcal{W})$ can be identified as

$$
\mathcal{F}_{\mathrm{b}}(\mathcal{W})=\mathcal{F}_{\mathrm{b}}\left(\mathcal{W}^{\prime}\right)=\mathcal{F}_{\mathrm{b}}\left(\mathcal{X}_{0}\right) \otimes \mathcal{F}_{\mathrm{b}}\left(\mathcal{X}_{0}^{\perp}\right)=\oplus_{n=0}^{\infty} \mathcal{G}_{n}
$$

with

$$
\mathcal{G}_{n}:=\mathcal{F}_{\mathrm{b}}^{(n)}\left(\mathcal{X}_{0}\right) \otimes \mathcal{F}_{\mathrm{b}}\left(\mathcal{X}_{0}^{\perp}\right)
$$

where

$$
\mathcal{F}_{\mathrm{b}}^{(n)}\left(\mathcal{X}_{0}\right) \cong\left\{z a\left(\phi_{0}\right)^{* n} \Omega_{\mathcal{W}} \mid z \in \mathbf{C}\right\} .
$$

It is easy to see that $H_{\mathrm{RWA}}$ identified with $d \Gamma(h)$ (Lemma 6.4) is reduced by each $\mathcal{G}_{n}$. We denote the reduced part by $H_{\mathrm{RWA}}^{(n)}$. Then we have

$$
\sigma\left(H_{\mathrm{RWA}}^{(n)}\right)=\left\{n x_{0}\right\} \cup\left[n x_{0}+\mu, \infty\right)
$$

where $n x_{0}$ is a simple eigenvalue of $H_{\mathrm{RWA}}^{(n)}$. This describes a more detailed structure of the specral properties stated in (ii) and (iii) of Theorem 6.9.

We now discuss some consequences of Theorem 6.9.

(1) Consider the case where $\mu=0$ and $\mu_{0}>\alpha^{2} \int_{\mathbf{R}^{d}} d k|\lambda(k)|^{2} / \omega(k)$ (hence $\mu_{0}>0$ ). Then Theorem 6.9(i) shows that $\bar{H}_{\text {RWA }}$ has a unique ground state $e_{0} \otimes \Omega$ even if $\langle\lambda, \omega\rangle$ obeys the infrared sigularity condition. If $\nu>0$ is sufficiently small, then $\mu_{0}>$ $\nu+\alpha^{2} \int_{\mathbf{R}^{d}} d k|\lambda(k)|^{2} / \omega(k)$. Hence, by Theorem 6.9(i), the regularized Hamiltonian $\bar{H}_{\mathrm{RWA}}(\nu)$ has a unique ground state $e_{0} \otimes \Omega$ with ground state-energy $E_{0}(\nu)=0$. Hence, in this case, $E_{0}(\nu)$ is trivially right differentiable at $\nu=0$ with $E_{0}^{\prime}(0+)=0$.

(2) Consider the case where $\mu=0$ and $\mu_{0}<\alpha^{2} \int_{\mathbf{R}^{d}} d k|\lambda(k)|^{2} / \omega(k)$ (this always holds if $\left.\mu_{0}<0\right)$. Then Theorem 6.9 (iii) shows that $\bar{H}_{\text {RWA }}$ has no ground states, although it has eigenvectors with nonpositive eigenvalues even if $\langle\lambda, \omega\rangle$ obeys the infrared singularity condition. 


\subsection{The Wigner-Weisskopf model}

This is a model of a two-level atom coupled to a quantized radiation field (e.g., [23] and references therein). It is obtained as a special simple realization of the GSB model with the following choice of $\left\{\mathcal{H}, A, B_{j}\right\}$ :,

$$
\mathcal{H}=\mathbf{C}^{2}, \quad J=1, \quad B_{1}=c:=\left(\begin{array}{ll}
0 & 0 \\
1 & 0
\end{array}\right), \quad A=\mu_{0} c^{*} c
$$

so that the Hamiltonian $H$ takes the form

$$
H_{\mathrm{ww}}:=\mu_{0} c^{*} c \otimes I+I \otimes d \Gamma(\hat{\omega})+\alpha\left(c^{*} \otimes a(\lambda)+c \otimes a(\lambda)^{*}\right),
$$

acting in the Hilbert space

$$
\mathcal{F}_{\text {WW }}:=\mathrm{C}^{2} \otimes \mathcal{F}_{\mathrm{b}}
$$

where $\mu_{0} \in \mathbf{R}$ is a constant parameter as in the RWA oscillator. Note that $c$ is a fermion annihilation operator of one degree, satisfying the canonical anticommutation relations

$$
c c^{*}+c^{*} c=I, \quad c^{2}=0=c^{* 2} .
$$

The Wigner-Weisskopf model may be regarded as the standard spin-boson model with a rotating wave approximation [35] exept for that $\mu_{0}$ is allowed to be nonpositive.

We continue to assume (6.2). Since $c$ is bounded (and hence $A=\mu_{0} c^{*} c$ too), this model trivially satisfies Hypothesis (H.2) for all $\alpha \in \mathbf{R} \backslash\{0\}$. Hence, by Proposition 2.1, for all $\alpha \in \mathbf{R} \backslash\{0\}, H_{\mathrm{ww}}$ is self-adjoint on $D\left(H_{\mathrm{ww}}\right)=D(I \otimes d \Gamma(\hat{\omega}))$ and bounded from below.

We have

$$
\mathcal{E}_{0}\left(\mu_{0} c^{*} c\right)=\frac{1}{2}\left(\mu_{0}-\left|\mu_{0}\right|\right) .
$$

Hence, by Proposition 2.3 and (2.27)

$$
\frac{1}{2}\left(\mu_{0}-\left|\mu_{0}\right|\right)-\alpha^{2}\left\|\frac{\lambda}{\sqrt{\omega}}\right\|_{L^{2}\left(\mathbf{R}^{d}\right)}^{2} \leq \mathcal{E}_{0}\left(H_{\mathrm{WW}}\right) \leq \frac{1}{2}\left(\mu_{0}-\left|\mu_{0}\right|\right) .
$$

A simple application of a theorem on the GSB model gives the following a priori information on the spectrum of $H_{\mathrm{Ww}}$.

Proposition 6.10 Assume (RWA).

(i) Let $\mu>0$. Then

$$
\sigma_{\mathrm{ess}}\left(H_{\mathrm{WW}}\right)=\left[\mathcal{E}_{0}\left(H_{\mathrm{WW}}\right)+\mu, \infty\right)
$$

and $H_{\mathrm{WW}}$ has purely discrete spectrum in $\left[\mathcal{E}_{0}\left(H_{\mathrm{Ww}}\right), \mathcal{E}_{0}\left(H_{\mathrm{Ww}}\right)+\mu\right)$.

(ii) Let $\mu=0$. Then

$$
\sigma\left(H_{\mathrm{WW}}\right)=\left[\mathcal{E}_{0}\left(H_{\mathrm{WW}}\right), \infty\right)
$$


Proof. See [14, Theorem 3.3, Remark 3.1] (cf. also [15]).

To analyze the spectral properties of $H_{\mathrm{ww}}$ in more detail, we show that the WignerWeisskopf model may be regarded as a "part" of the RWA oscillator. We first indentify $\mathcal{F}_{\text {RWA }}$ as

$$
\mathcal{F}_{\mathrm{RWA}}=\oplus_{n=0}^{\infty} \mathcal{F}_{\mathrm{b}}=\left\{\Psi=\left\{\Psi^{(n)}\right\}_{n=0}^{\infty} \mid \Psi^{(n)} \in \mathcal{F}_{\mathrm{b}}, n \geq 0, \sum_{n=0}^{\infty}\left\|\Psi^{(n)}\right\|_{\mathcal{F}_{\mathrm{b}}}^{2}<\infty\right\}
$$

such that $(b \otimes I) \Psi$ is identified with $\left\{\sqrt{n+1} \Psi^{(n+1)}\right\}_{n=0}^{\infty}$ and, for a linear operator $T$ on $\mathcal{F}_{\mathrm{b}},(I \otimes T) \Psi$ is identified with $\left\{T \Psi^{(n)}\right\}_{n=0}^{\infty}$.

The Hilbert space $\mathcal{F}_{\mathrm{WW}}$ can be identified as

$$
\mathcal{F}_{\mathrm{WW}}=\mathcal{F}_{\mathrm{b}} \oplus \mathcal{F}_{\mathrm{b}}=\left\{\Phi=\left\langle\Phi^{(0)}, \Phi^{(1)}\right\rangle \mid \Phi^{(s)} \in \mathcal{F}_{\mathrm{b}}, s=0,1\right\}
$$

where the operator $M \otimes I$ on $\mathcal{F}_{\text {WW }}$ with a $2 \times 2$ matrix $M=\left(M_{r s}\right)_{r, s=0,1}$ is identified with the operator $M$ acting as

$$
M \Phi=\left(\begin{array}{ll}
M_{00} & M_{01} \\
M_{10} & M_{11}
\end{array}\right)\left(\begin{array}{l}
\Phi^{(1)} \\
\Phi^{(0)}
\end{array}\right)
$$

Note that the convention for writing the column vectors is different from the usual one: the row vector $\left\langle\Phi^{(0)}, \Phi^{(1)}\right\rangle$ is identified with the column vector $\left(\begin{array}{l}\Phi^{(1)} \\ \Phi^{(0)}\end{array}\right)$. With these idetifications, we define an operator $V: \mathcal{F}_{\text {RWA }} \rightarrow \mathcal{F}_{\text {WW }}$ by

$$
V \Psi:=\left\langle\Psi^{(0)}, \Psi^{(1)}\right\rangle, \quad \Psi=\left\{\Psi^{(n)}\right\}_{n=0}^{\infty} \in \mathcal{F}_{\mathrm{RWA}} .
$$

It is easy to see that $V$ is a partial isometry:

$$
V V^{*}=I, \quad V^{*} V=P
$$

where $P$ is the orthogonal projection from $\mathcal{F}_{\text {RWA }}$ onto the closed subspace

$$
\left\{\Psi=\left\{\Psi^{(n)}\right\}_{n=0}^{\infty} \in \mathcal{F}_{\mathrm{RWA}} \mid \Psi^{(n)}=0 \text { for all } n \geq 2\right\} .
$$

A key fact is the following:

Lemma 6.11 The operator equation

$$
V H_{\mathrm{RWA}} V^{*}=H_{\mathrm{WW}}
$$

holds. In particular,

$$
\mathcal{E}_{0}\left(H_{\mathrm{WW}}\right) \geq \mathcal{E}_{0}\left(\bar{H}_{\mathrm{RWA}}\right)
$$


Proof. We first show by direct computation that each $\Phi \in D\left(H_{\mathrm{Ww}}\right)=D(d \Gamma(\hat{\omega})) \oplus$ $D(d \Gamma(\hat{\omega}))$ is in $D\left(V H_{\mathrm{RWA}} V^{*}\right)$ and $V H_{\mathrm{RWA}} V^{*} \Phi=H_{\mathrm{WW}} \Phi$. This fact and the self-adjointness of $H_{\mathrm{WW}}$ imply the operator equation (6.46). Inequality (6.47) is a direct consequence of (6.46) and the variational principle.

Let

$$
N:=c^{*} c \otimes I+I \otimes N_{\mathrm{b}}
$$

Lemma 6.12 $N$ strongly commutes with $H_{\mathrm{WW}}$.

Proof. Let $\Psi \in D\left(H_{W W}\right) \cap\left(\mathbf{C}^{2} \otimes \mathcal{F}_{\mathrm{b}, 0}\right)$ (for $\mathcal{F}_{\mathrm{b}, 0}$, see the proof of Proposition 2.3). Then we have for all $t \in \mathbf{R}$

$$
\begin{aligned}
& e^{i t N}(c \otimes I) e^{-i t N} \Psi=e^{-i t}(c \otimes I), \quad e^{i t N}\left(c^{*} \otimes I\right) e^{-i t N} \Psi=e^{i t}\left(c^{*} \otimes I\right), \\
& e^{i t N}(I \otimes a(f)) e^{-i t N} \Psi=e^{-i t} I \otimes a(f) \Psi, \\
& e^{i t N}\left(I \otimes a(f)^{*}\right) e^{-i t N} \Psi=e^{i t} I \otimes a(f)^{*} \Psi, \quad f \in L^{2}\left(\mathbf{R}^{d}\right) .
\end{aligned}
$$

Hence it follows that

$$
e^{i t N} H_{\mathrm{WW}} \Psi=H_{\mathrm{WW}} e^{i t N} \Psi
$$

Since $D\left(H_{W W}\right) \cap\left(\mathbf{C}^{2} \otimes \mathcal{F}_{\mathrm{b}, 0}\right)$, is a core of $H_{\mathrm{WW}}$, we obtain that $e^{i t N} H_{\mathrm{WW}} \subset H_{\mathrm{WW}} e^{i t N}$, which implies the the strong commutativity of $N$ and $H_{\mathrm{ww}}$.

The spectrum of $N$ consists of only eigenvalues with

$$
\sigma(N)=\sigma_{\mathrm{p}}(N)=\{0,1,2, \cdots\} .
$$

The eigenspace of $N$ with the eigenvalue $\ell$ is

$$
\mathcal{F}_{\mathrm{WW}}^{(\ell)}:=\left\{\left\langle\Psi^{(\ell)}, \Phi^{(\ell-1)}\right\rangle \mid \Psi^{(\ell)} \in \mathcal{F}_{\mathrm{b}}^{(\ell)}, \Phi^{(\ell-1)} \in \mathcal{F}_{\mathrm{b}}^{(\ell-1)}\right\}
$$

where $\mathcal{F}_{\mathrm{b}}^{(\ell)}$ is the $\ell$-particle space of $\mathcal{F}_{\mathrm{b}}: N_{\mathrm{b}} \mid \mathcal{F}^{(\ell)}=\ell$ and $\Phi^{(-1)}:=\{0\}$. We have

$$
\mathcal{F}_{\mathrm{WW}}=\oplus_{\ell=0}^{\infty} \mathcal{F}_{\mathrm{WW}}^{(\ell)} .
$$

By Lemma $6.12, H_{\mathrm{WW}}$ is reduced by each $\mathcal{F}_{\mathrm{WW}}^{(\ell)}$. We denote the reduced part by $H_{\mathrm{WW}}^{(\ell)}$. Then we have

$$
H_{\mathrm{WW}}=\oplus_{\ell=0}^{\infty} H_{\mathrm{WW}}^{(\ell)}
$$

with respect to the decomposition (6.49). It is easy to see that

$$
H_{\mathrm{WW}}^{(0)}=0
$$

Every vector $\Xi \in \mathcal{F}_{\mathrm{WW}}^{(1)}$ has the form

$$
\Xi=\left\langle a(f)^{*} \Omega, z \Omega\right\rangle
$$


with $z \in \mathbf{C}, f \in L^{2}\left(\mathbf{R}^{d}\right)$. By direct computation, we have

$$
H_{\mathrm{WW}}^{(1)} \Xi=\left\langle a(\omega f+z \lambda)^{*} \Omega,\left(z+\alpha(\lambda, f)_{L^{2}\left(\mathbf{R}^{d}\right)}\right) \Omega\right\rangle .
$$

It is easy to see that the operator $U_{1}: \mathcal{F}_{\mathrm{WW}}^{(1)} \rightarrow \mathcal{W}=\mathbf{C} \oplus L^{2}\left(\mathbf{R}^{d}\right)$ defined by

$$
U_{1} \Xi:=\langle z, f\rangle
$$

is unitary. By (6.52), we see that

$$
U_{1} H_{\mathrm{WW}}^{(1)} U_{1}^{-1}=h
$$

Thus

$$
\sigma\left(H_{\mathrm{WW}}^{(1)}\right)=\sigma(h), \quad \sigma_{\mathrm{p}}\left(H_{\mathrm{WW}}^{(1)}\right)=\sigma_{\mathrm{p}}(h) .
$$

Proposition 6.13 (i) Let $d_{\mu} \geq 0$. Then 0 is a simple eigenvalue of $H_{\mathrm{WW}}$ with an eigenvector

$$
\Psi_{0}:=\langle\Omega, 0\rangle .
$$

Moreover, in the case $\mu>0, H_{\mathrm{Ww}}$ has no eigenvalues in the open interval $(0, \mu)$.

(ii) Let $d_{\mu}<0$ and $\mu_{0} \neq \alpha^{2}\|\lambda / \sqrt{\omega}\|_{L^{2}\left(\mathbf{R}^{d}\right)}^{2}$. Then 0 is a eigenvalue of $H_{\mathrm{WW}}$ with an eigenvector $\Psi_{0}$ and $x_{0}$ is a non-zero eigenvalue of $H_{\mathrm{Ww}}$ with an eigenvector

$$
\Phi_{0}:=\left\langle-\alpha a\left(\left(\omega-x_{0}\right)^{-1} \lambda\right)^{*} \Omega, \Omega\right\rangle .
$$

If $\mu_{0}>\alpha^{2}\|\lambda / \sqrt{\omega}\|_{L^{2}\left(\mathbf{R}^{d}\right)}^{2}$ (resp. $\left.\mu_{0}<\alpha^{2}\|\lambda / \sqrt{\omega}\|_{L^{2}\left(\mathbf{R}^{d}\right)}^{2}\right)$, then $x_{0}>0$ (resp. $\left.x_{0}<0\right)$.

(iii) Let $d_{\mu}<0$ and $\mu_{0}=\alpha^{2}\|\lambda / \sqrt{\omega}\|_{L^{2}\left(\mathbf{R}^{d}\right)}^{2}$. Let $\lambda / \omega \in L^{2}\left(\mathbf{R}^{d}\right)$. Then 0 is a degenerate eigenvalue of $H_{\mathrm{WW}}$ with multiplicity more than or equal to two, two independent eigenvectors (up to constant multiples) being $\Psi_{0}$ and

$$
\Xi_{0}:=\left\langle-\alpha a\left(\omega^{-1} \lambda\right)^{*} \Omega, \Omega\right\rangle
$$

Proof. (i) It is straightforward to see that $H_{\mathrm{WW}} \Psi_{0}=0$. To prove the simplicity of the eigenvalue 0 , let $H_{\mathrm{WW}} \Theta=0$ with $\Theta=\left\{\Theta^{(\ell)}\right\}_{\ell=0}^{\infty} \in D\left(H_{\mathrm{WW}}\right), \Theta^{(\ell)}=\left\langle\Psi^{(\ell)}, \Phi^{(\ell-1)}\right\rangle$. Then

$$
\begin{aligned}
& \left(H_{\mathrm{b}}+\mu_{0}\right) \Phi^{(\ell-1)}=-\alpha a(\lambda) \Psi^{(\ell)}, \\
& H_{\mathrm{b}} \Psi^{(\ell)}=-\alpha a(\lambda)^{*} \Phi^{(\ell-1)},
\end{aligned}
$$

where

$$
H_{\mathrm{b}}:=d \Gamma(\hat{\omega})
$$

The condition $d_{\mu} \geq 0$ gives

$$
\mu_{0}-\mu \geq \alpha^{2} \int_{\mathbf{R}^{d}} \frac{|\lambda(k)|^{2}}{\omega(k)-\mu} d k
$$


In particular, $\mu_{0} \geq \mu>0$. Let $\ell \geq 2$. Then, by (6.58), we have

$$
\Psi^{(\ell)}=-\alpha H_{\mathrm{b}}^{-1} a(\lambda) \Phi^{(\ell-1)} .
$$

Putting this into (6.57), we obtain

$$
\left(H_{\mathrm{b}}+\mu_{0}\right) \Phi^{(\ell-1)}=\alpha^{2} a(\lambda) H_{\mathrm{b}}^{-1} a(\lambda)^{*} \Phi^{(\ell-1)} .
$$

Hence

$$
\begin{aligned}
\left(\mu(\ell-1)+\mu_{0}\right)\left\|\Phi^{(\ell-1)}\right\|^{2} & \leq\left(\Phi^{(\ell-1)},\left(H_{\mathrm{b}}+\mu_{0}\right) \Phi^{(\ell-1)}\right) \\
& =\alpha^{2}\left(\Phi^{(\ell-1)}, a(\lambda) H_{\mathrm{b}}^{-1} a(\lambda)^{*} \Phi^{(\ell-1)}\right) .
\end{aligned}
$$

Inequality (6.60) implies that

$$
\alpha^{2} a(\lambda)^{*} a(l a) \leq\left(\mu_{0}-\mu\right)\left(H_{\mathrm{b}}-\mu N_{\mathrm{b}}\right),
$$

leading to

$$
\alpha^{2}\left(\Psi^{(\ell-1)}, a(\lambda) H_{\mathrm{b}}^{-1} a(\lambda)^{*} \Phi^{(\ell-1)}\right) \leq\left(\mu_{0}-\mu\right)\left\|\Phi^{(\ell-1)}\right\|^{2}
$$

(see [35, p.319]). Hence, if $\Phi^{(\ell-1)} \neq 0$, then

$$
\mu(\ell-1)+\mu_{0} \leq \mu_{0}-\mu .
$$

But this is a contradiction. Hence, for all $\ell \geq 2, \Phi^{(\ell-1)}=0$ and hence $\Psi^{(\ell)}=0$. Thus $\Theta^{(\ell)}=0, \ell \geq 2$. By (6.53) and Theorem 6.8(i), $\Theta^{(1)}=0$. Thus $\Theta$ is a constant multiple of $\Psi_{0}$.

Let $\mu>0$ and suppose that $H_{\mathrm{WW}}$ had an eigenvalue $E$ in $(0, \mu)$ with an eigenvector $\Theta=\left\{\Theta^{(\ell)}\right\}_{\ell=0}^{\infty}(\neq 0) \in D\left(H_{\mathrm{WW}}\right), \Theta^{(\ell)}=\left\langle\Psi^{(\ell)}, \Phi^{(\ell-1)}\right\rangle$. Then, for all $\ell \geq 0$,

$$
\begin{aligned}
& \left(H_{\mathrm{b}}+\mu_{0}-E\right) \Phi^{(\ell-1)}=-\alpha a(\lambda) \Psi^{(\ell)}, \\
& \left(H_{\mathrm{b}}-E\right) \Psi^{(\ell)}=-\alpha a(\lambda)^{*} \Phi^{(\ell-1)} .
\end{aligned}
$$

Since $E \in \varrho\left(H_{\mathrm{b}}\right)$, we have by $(6.62)$

$$
\Psi^{(\ell)}=-\alpha\left(H_{\mathrm{b}}-E\right)^{-1} a(\lambda)^{*} \Phi^{(\ell-1)} .
$$

Putting this into (6.61), we obtain

$$
\left(H_{\mathrm{b}}+\mu_{0}-E\right) \Phi^{(\ell-1)}=\alpha^{2} a(\lambda)\left(H_{\mathrm{b}}-E\right)^{-1} a(\lambda)^{*} \Phi^{(\ell-1)} .
$$

In the same way as above, one can show, using this equation, that, if $\Phi^{(\ell-1)} \neq 0$, then $(\ell-1) \mu+\mu_{0}-E \leq \mu_{0}-\mu$, i.e., $\ell \mu \leq E$. Hence, for all $\ell \geq 1, \Phi^{(\ell-1)}=0$ and hence $\Psi^{(\ell)}=0$. It is easy to see that $\Psi^{(0)}=0$. Hence $\Theta=0$. But this is a contradiction. Thus $E$ cannot be an eigenvalue of $H_{\mathrm{Ww}}$.

(ii) An easy exercise.

(iii) Note that, if $\mu_{0}=\alpha^{2}\|\lambda / \sqrt{\omega}\|_{L^{2}\left(\mathbf{R}^{d}\right)}^{2}$, then $x_{0}=0$. It is easy to see that $H_{\mathrm{WW}} \Xi_{0}=0$. 
Theorem 6.14 Let the same assumption as in Theorem 6.8 be satisfied.

(i) Let $d_{\mu} \geq 0$. Then

$$
\begin{aligned}
& \sigma_{\mathrm{ess}}\left(H_{\mathrm{WW}}\right)=[\mu, \infty), \\
& 0 \in \sigma_{\mathrm{p}}\left(H_{\mathrm{WW}}\right),
\end{aligned}
$$

where 0 is a simple eigenvalue with an eigenvector $\Psi_{0}$. In particular, $H_{\mathrm{WW}}$ is nonnegative with ground-state energy $\mathcal{E}_{0}\left(H_{\mathrm{WW}}\right)=0$. If $\mu>0$ in addition,

$$
\sigma_{\mathrm{p}}\left(H_{\mathrm{WW}}\right) \cap[0, \mu)=\{0\} .
$$

(ii) Let $d_{\mu}<0, \mu>0$ and $\mu_{0} \geq \alpha^{2}\|\lambda / \sqrt{\omega}\|_{L^{2}\left(\mathbf{R}^{d}\right)}^{2}$. Then,

$$
\begin{aligned}
& \sigma_{\mathrm{ess}}\left(H_{\mathrm{WW}}\right)=[\mu, \infty), \\
& \left\{0, x_{0}\right\} \subset \sigma_{\mathrm{p}}\left(H_{\mathrm{WW}}\right),
\end{aligned}
$$

with $0 \leq x_{0}<\mu$. In particular, $H_{\mathrm{WW}}$ is nonnegative with $\mathcal{E}_{0}\left(H_{\mathrm{WW}}\right)=0$.

Proof. (i) By Theorem 6.9(i) and (6.47), $H_{\mathrm{Ww}} \geq 0$. This fact and Proposition 6.13(i) imply that $\mathcal{E}_{0}\left(H_{\mathrm{WW}}\right)=0$. Hecne, by Proposition 6.10 , we obtain (6.63). (6.64) and (6.65) follow from Proposition 6.13(i).

(ii) Similar to part (i).

In the case

$$
\mu_{0}<\alpha^{2}\left\|\frac{\lambda}{\sqrt{\omega}}\right\|_{L^{2}\left(\mathbf{R}^{d}\right)}^{2}
$$

hence $\left.d_{\mu}<0\right)$, some difficulty arises in determining $\mathcal{E}_{0}\left(H_{\mathrm{WW}}\right)$ explicitly, because, in this case, $H_{\text {RWA }}$ is unbounded below (Theorem 6.9(iii)) and hence one cannot make use of Lemma 6.11. As for this problem, we present only a partial solution below.

Under condition (6.68), we can define

$$
M\left(\alpha, \mu_{0}, \omega\right):=\int_{\mathbf{R}^{d}} \frac{|\lambda(k)|^{2}}{\omega(k)-\mu_{0}+\alpha^{3}\|\lambda / \sqrt{\omega}\|_{L^{2}\left(\mathbf{R}^{d}\right)}^{2}} d k .
$$

Theorem 6.15 Let $\mu_{0}<0$ (hence (6.68) trivially holds) and

$$
2 \mu-\mu_{0}>\alpha^{2}\left(\left\|\frac{\lambda}{\sqrt{\omega}}\right\|_{L^{2}\left(\mathbf{R}^{d}\right)}^{2}-M\left(\alpha, \mu_{0}, \omega\right)\right)+\frac{\|\lambda\|_{L^{2}\left(\mathbf{R}^{d}\right)}^{2}}{M\left(\alpha, \mu_{0}, \omega\right)} .
$$

Then

$$
\mathcal{E}_{0}\left(H_{\mathrm{WW}}\right)=x_{0}
$$


Proof. By Proposition 6.13(ii),

$$
E:=\mathcal{E}_{0}\left(H_{\mathrm{WW}}\right) \leq x_{0}<\mu_{0}<0 .
$$

We first consider the case $\mu>0$. Then, by Proposition 6.10(i), $E$ is an eigenvalue of $H_{\mathrm{WW}}$. Hence $H_{\mathrm{WW}}$ has a ground state $\Theta_{0}=\left\{\Theta_{0}^{(\ell)}\right\}_{\ell=0}^{\infty} \in D\left(H_{\mathrm{WW}}\right)$ satisfying $H_{\mathrm{WW}} \Theta_{0}=E \Theta_{0}$, which implies that, for all $\ell \geq 0$,

$$
H_{\mathrm{WW}}^{(\ell)} \Theta_{0}^{(\ell)}=E \Theta_{0}^{(\ell)}
$$

By (6.51) and (6.72), $\Theta_{0}^{(0)}=0$. If $\Theta_{0}^{(1)} \neq 0$, then, by Proposition 6.13(ii) and (6.72), we obtain (6.71). Therefore we consider the case $\Theta_{0}^{(1)}=0$. Then, for some $\ell \geq 2, \Theta_{0}^{(\ell)} \neq 0$. Let $\Theta_{0}^{(\ell)}=\left\langle\Psi^{(\ell)}, \Phi^{(\ell-1)}\right\rangle$. Then (6.73) is equivalent to (6.61) and (6.62). By (6.61) and the property $\mu_{0}-E>0$, we have

$$
\Phi^{(\ell-1)}=-\alpha\left(H_{\mathrm{b}}+\mu_{0}-E\right)^{-1} a(\lambda) \Psi^{(\ell)} .
$$

Putting this into (6.62), we obtain

$$
\left(H_{\mathrm{b}}-E\right) \Psi^{(\ell)}=\alpha^{2} a(\lambda)^{*}\left(H_{\mathrm{b}}+\mu_{0}-E\right)^{-1} a(\lambda) \Psi^{(\ell)} .
$$

Hence

$$
\left(\Psi^{(\ell)},\left(H_{\mathrm{b}}-E\right) \Psi^{(\ell)}=\alpha^{2}\left\|\left(H_{\mathrm{b}}+\mu_{0}-E\right)^{-1 / 2} a(\lambda) \Psi^{(\ell)}\right\|^{2},\right.
$$

which implies that

$$
\left.(\mu \ell-E)\left\|\Psi^{(\ell)}\right\|^{2} \leq \alpha^{2} \| H_{\mathrm{b}}+\mu_{0}-E\right)^{-1 / 2} a(\lambda) \Psi^{(\ell)} \|^{2} .
$$

To estimate the right hand side, we note that $\left(H_{\mathrm{b}}+\mu_{0}-E\right)^{-1 / 2} a(\lambda)$ is bounded with

$$
\left[\left(H_{\mathrm{b}}+\mu_{0}-E\right)^{-1 / 2} a(\lambda)\right]^{*}=a(\lambda)^{*}\left(H_{\mathrm{b}}+\mu_{0}-E\right)^{-1 / 2}
$$

and, by $(4.23)$,

$$
\left\|a(\lambda)^{*}\left(H_{\mathrm{b}}+\mu_{0}-E\right)^{-1 / 2} \Psi^{(\ell)}\right\|^{2} \leq\left\|\frac{\lambda}{\sqrt{\omega}}\right\|_{L^{2}\left(\mathbf{R}^{d}\right)}^{2}\left\|\Psi^{(\ell)}\right\|^{2}+\frac{\|\lambda\|_{L^{2}\left(\mathbf{R}^{d}\right)}^{2}}{\mu \ell+\mu_{0}-E}\left\|\Psi^{(\ell)}\right\| .
$$

Hence

$$
\mu \ell-\alpha^{2}\left\|\frac{\lambda}{\sqrt{\omega}}\right\|_{L^{2}\left(\mathbf{R}^{d}\right)}^{2}-\alpha^{2} \frac{\|\lambda\|_{L^{2}\left(\mathbf{R}^{d}\right)}^{2}}{\mu \ell+\mu_{0}-E} \leq E,
$$

where we have used that $\Psi^{(\ell)} \neq 0$ (if $\Psi^{(\ell)}=0$, then $\Phi^{(\ell-1)}=0$ by (6.74) and hence $\Theta_{0}^{(\ell)}=0$, but this is a contradiction). An application of (2.21) yields

$$
E \geq \mu_{0}-\alpha^{2}\left\|\frac{\lambda}{\sqrt{\omega}}\right\|_{L^{2}\left(\mathbf{R}^{d}\right)}^{2}
$$


Now suppose that $E<x_{0}$. Then $D(E)>D\left(x_{0}\right)=0$, i.e.,

$$
E<\mu_{0}-\alpha^{2} \int_{\mathbf{R}^{d}} \frac{|\lambda(k)|^{2}}{\omega(k)-E} d k
$$

which, combined with (6.75), gives

$$
\begin{aligned}
\mu \ell-\mu_{0} & <\alpha^{2}\left\|\frac{\lambda}{\sqrt{\omega}}\right\|_{L^{2}\left(\mathbf{R}^{d}\right)}^{2}+\alpha^{2} \frac{\|\lambda\|_{L^{2}\left(\mathbf{R}^{d}\right)}^{2}}{\mu \ell+\mu_{0}-E}-\alpha^{2} \int_{\mathbf{R}^{d}} \frac{|\lambda(k)|^{2}}{\omega(k)-E} d k \\
& <\alpha^{2}\left(\left\|\frac{\lambda}{\sqrt{\omega}}\right\|_{L^{2}\left(\mathbf{R}^{d}\right)}^{2}-M\left(\alpha, \mu_{0}, \omega\right)\right)+\frac{\|\lambda\|_{L^{2}\left(\mathbf{R}^{d}\right)}^{2}}{M\left(\alpha, \mu_{0}, \omega\right)} .
\end{aligned}
$$

Since $\ell \geq 2,(6.77)$ implies that

$$
2 \mu-\mu_{0}<\alpha^{2}\left(\left\|\frac{\lambda}{\sqrt{\omega}}\right\|_{L^{2}\left(\mathbf{R}^{d}\right)}^{2}-M\left(\alpha, \mu_{0}, \omega\right)\right)+\frac{\|\lambda\|_{L^{2}\left(\mathbf{R}^{d}\right)}^{2}}{M\left(\alpha, \mu_{0}, \omega\right)} .
$$

But this contradicts (6.70). Thus (6.71) holds.

We next consider the case $\mu=0$. Let $\nu>0$ and

$$
H_{\mathrm{wW}}(\nu):=\mu_{0} c^{*} c \otimes I+I \otimes d \Gamma\left(\hat{\omega}_{\nu}\right)+\alpha\left(c^{*} \otimes a(\lambda)+c \otimes a(\lambda)^{*}\right),
$$

the regularized Wigner-Weisskopf Hamiltonian. Then, by Proposition 3.2(iii),

$$
\lim _{\nu \rightarrow 0} \mathcal{E}_{0}\left(H_{\mathrm{WW}}(\nu)\right)=\mathcal{E}_{0}\left(H_{\mathrm{WW}}\right) .
$$

For all sufficiently small $\nu>0$, condition (6.70) with $\omega$ replaced by $\omega_{\nu}$ is satisfied. Hence, by the above result on $\mathcal{E}_{0}\left(H_{\mathrm{WW}}\right)$ with $\mu>0$, we have $\mathcal{E}_{0}\left(H_{\mathrm{Ww}}(\nu)\right)=x_{0}(\nu)$ for all sufficiently small $\nu>0$, where $x_{0}(\nu)$ is the $x_{0}$ with $\omega$ replaced by $\omega_{\nu}$. It is easy to see that $\lim _{\nu \rightarrow 0} x_{0}(\nu)=x_{0}$. Hence (6.71) follows.

We now discuss consequences of Theorems 6.14 and 6.15 in view of the right differntiability of the ground-state energy of the regularized model. Hence we consider the case $\mu=0$ and the regularized Hamiltonian $H_{\mathrm{Ww}}(\nu)$ of the Wigner-Weisskopf model given by (6.78). We denote its ground-state energy by $E_{\mathrm{Ww}, 0}(\nu)$.

(1) Consider the case $\mu=0$ and let $d_{0}>0$, i.e., $\mu_{0}>\alpha^{2} \int_{\mathbf{R}^{d}} d k|\lambda(k)|^{2} / \omega(k)$ (hence $\left.\mu_{0}>0\right)$. Then Theorem 6.14(i) shows that $H_{\mathrm{WW}}$ has a unique ground state $\langle\Omega, 0\rangle$ with $E_{\mathrm{WW}, 0}(0)=0$ even if $\langle\lambda, \omega\rangle$ obeys the infrared sigularity condition. If $\nu>0$ is sufficiently small, then $\mu_{0}>\nu+\alpha^{2} \int_{\mathbf{R}^{d}} d k|\lambda(k)|^{2} / \omega(k)$. Hence, by Theorem 6.14(i), $H_{\mathrm{WW}}(\nu)$ has a unique ground state $\langle\Omega, 0\rangle$ with $E_{\mathrm{WW}, 0}(\nu)=0$. Hence, in this case, $E_{\mathrm{WW}, 0}(\nu)$ is trivially right differentiable at $\nu=0$ with $E_{\mathrm{WW}, 0}^{\prime}(0+)=0$. 
(2) Consider the case $\mu=0$ and $\mu_{0}<0$. Let

$$
-\mu_{0}>\alpha^{2}\left(\left\|\frac{\lambda}{\sqrt{\omega}}\right\|_{L^{2}\left(\mathbf{R}^{d}\right)}^{2}-M\left(\alpha, \mu_{0}, \omega\right)\right)+\frac{\|\lambda\|_{L^{2}\left(\mathbf{R}^{d}\right)}^{2}}{M\left(\alpha, \mu_{0}, \omega\right)} .
$$

Then

$$
2 \nu-\mu_{0}>\alpha^{2}\left(\left\|\frac{\lambda}{\sqrt{\omega_{\nu}}}\right\|_{L^{2}\left(\mathbf{R}^{d}\right)}^{2}-M\left(\alpha, \mu_{0}, \omega_{\nu}\right)\right)+\frac{\|\lambda\|_{L^{2}\left(\mathbf{R}^{d}\right)}^{2}}{M\left(\alpha, \mu_{0}, \omega_{\nu}\right)} .
$$

for all sufficiently small $\nu>0$. We consider only such $\nu$ 's. Theorem 6.15 shows that $H_{\mathrm{WW}}$ has a ground state $\Phi_{0}$ with $E_{\mathrm{WW}, 0}(0)=x_{0}$ even if $\langle\lambda, \omega\rangle$ obeys the infrared sigularity condition. Similarly $H_{\mathrm{WW}}(\nu)$ has a ground state with ground-state energy $E_{\mathrm{WW}, 0}(\nu)<0$ satisfying

$$
E_{\mathrm{WW}, 0}(\nu)=\mu_{0}+\alpha^{2} \int_{\mathbf{R}^{d}} \frac{|\lambda(k)|^{2}}{E_{\mathrm{WW}, 0}(\nu)-\omega(k)-\nu} d k .
$$

Hence we have

$$
\frac{E_{\mathrm{WW}, 0}(\nu)-E_{\mathrm{WW}, 0}(0)}{\nu}=\frac{\Lambda(\nu)}{1+\Lambda(\nu)}
$$

with

$$
\Lambda(\nu):=\alpha^{2} \int_{\mathbf{R}^{d}} d k \frac{|\lambda(k)|^{2}}{\left(E_{\mathrm{WW}, 0}(\nu)-\omega(k)-\nu\right)\left(E_{\mathrm{WW}, 0}(0)-\omega(k)\right)} .
$$

Since $E_{\mathrm{WW}, 0}(\nu)<0$, it follows that

$$
\Lambda_{0}:=\lim _{\nu \downarrow 0} \Lambda(\nu)=\alpha^{2} \int_{\mathbf{R}^{d}} d k \frac{|\lambda(k)|^{2}}{\left(E_{\mathrm{WW}, 0}(0)-\omega(k)\right)^{2}} .
$$

Hence $E_{\mathrm{WW}, 0}(\nu)$ is right differentiable at $\nu=0$ with

$$
E_{\mathrm{WW}, 0}^{\prime}(0+)=\frac{\Lambda_{0}}{1+\Lambda_{0}}<1
$$

Let $\Phi_{0}(\nu)$ be the vector $\Phi_{0}$ with $\omega$ replaced by $\omega_{\nu}$. Then the ground-state expectation of the number operator is computed as

$$
n_{\mathrm{wW}}(\nu):=\left(\Phi_{0}(\nu), N_{\mathrm{b}} \Phi_{0}(\nu)\right)=\alpha^{2}\left\|\left(\omega-E_{\mathrm{WW}, 0}(\nu)\right)^{-1} \lambda\right\|_{L^{2}\left(\mathbf{R}^{d}\right)}^{2}<\infty .
$$




\section{Appendix}

\section{A Some General Properties of the Ground-State En- ergy for a Class of Self-Adjoint Operators}

Let $\mathcal{K}$ be a Hilbert space and $T$ (resp. $S$ ) be a self-adjoint (resp. symmetric) operator on $\mathcal{K}$. We suppose that $T$ is bounded from below. For $\nu \geq 0$, we define

$$
T(\nu):=T+\nu S
$$

We assume the following:

(T.1) There exists a constant $c>0$ such that, for all $\nu \in(0, c), T(\nu)$ is self-adjoint on $D(T) \cap D(S)$ and bounded from below.

Under this hypothesis, we can define

$$
G(\nu):=\mathcal{E}_{0}(T(\nu)):=\inf \sigma(T(\nu)), \quad \nu \in[0, c)
$$

the ground-state energy of $T(\nu)$. The following proposition summarizes elementary properties of $G(\nu)$.

Proposition A.1 Assume (T.1).

(i) If $S \geq 0$, then $G(\nu)$ is monotone nondecreasing in $\nu \in[0, c)$.

(ii) The function $G(\nu)$ is concave, i.e., for all $\nu, \nu^{\prime} \in[0, c)$ and $t \in[0,1]$,

$$
t G(\nu)+(1-t) G\left(\nu^{\prime}\right) \leq G\left(t \nu+(1-t) \nu^{\prime}\right)
$$

(iii) Let $a \in(0, c)$ be a constant such that $\inf _{m \in[0, a]} G(\nu)>-\infty$. Then $G(\nu)$ is continuous on $(0, a)$. Moreover, for all $\nu \in(0, a)$,

$$
G^{\prime}(\nu \pm 0):=\lim _{\varepsilon \downarrow 0} \frac{G(\nu \pm \varepsilon)-G(\nu)}{ \pm \varepsilon}
$$

exist and

$$
G^{\prime}(\nu+0) \leq G^{\prime}(\nu-0)
$$

(iv) If $S \geq 0$, then $G(\nu)$ is continuous on $(0, c)$ and, for all $\nu \in(0, c), G^{\prime}(\nu \pm 0)$ exist, satisfying (A.5). Moreover, if $D(T) \cap D(S)$ is a core of $T$ in addition, then $G(\nu)$ is right continuous at $m=0$. 
Proof. (i) Let $0 \leq \nu<\nu^{\prime}<c$. Then

$$
T(\nu)=T\left(\nu^{\prime}\right)-\left(\nu^{\prime}-\nu\right) S
$$

Hence, for all $\Psi \in D\left(T\left(\nu^{\prime}\right)\right)=D(T) \cap D(S)$ with $\|\Psi\|=1$,

$$
G(\nu) \leq(\Psi, T(\nu) \Psi)=\left(\Psi, T\left(\nu^{\prime}\right) \Psi\right)-\left(\nu^{\prime}-\nu\right)(\Psi, S \Psi) .
$$

By the assumption $S \geq 0$, the second term on the right hand side is nonpositive. Hence $G(\nu) \leq\left(\Psi, T\left(\nu^{\prime}\right) \Psi\right)$, which implies that $G(\nu) \leq G\left(\nu^{\prime}\right)$. Thus $G(\nu)$ is nondecreasing in $\nu$.

(ii) We have for all $\nu, \nu^{\prime} \in[0, c)$

$$
T\left(t \nu+(1-t) \nu^{\prime}\right)=t T(\nu)+(1-t) T\left(\nu^{\prime}\right)
$$

Hence, by the variational principle, $t G(\nu)+(1-t) G\left(\nu^{\prime}\right) \leq T\left(t \nu+(1-t) \nu^{\prime}\right)$. By this inequality and the variational principle again, we obtain (A.3).

(iii) The continuity of $G(\nu)$ on $(0, a)$ and (A.5) follow from (ii) and a general theorem on concave functions.

(iv) In this case, by part (i), we have $G(\nu) \geq G(0), \nu \in(0, c)$. Hence $\inf _{\nu \in[0, c)} G(\nu)$ $\geq G(0)>-\infty$. Thus we can apply (iii) to conclude that $G(\nu)$ is continuous on $(0, c)$ and that, for all $\nu \in(0, c), G^{\prime}(\nu \pm 0)$ exist, satisfying (A.5). It remains to prove the right continuity of $G(\nu)$ at $\nu=0$ under the additional condition that $D(T) \cap D(S)$ is a core of $T$. By part (i), we have $G(0) \leq G(\nu), \nu \in(0, c)$. Hence $G(0) \leq \liminf _{\nu \downarrow 0} G(\nu)$. On the other hand, we have for all $\Psi \in D(T) \cap D(S)$ with $\|\Psi\|=1$

$$
G(\nu) \leq(\Psi, T \Psi)+\nu(\Psi, S \Psi)
$$

Hence $\lim \sup _{\nu \downarrow 0} G(\nu) \leq(\Psi, T \Psi)$. Since $D(T) \cap D(S)$ is a core of $T$, this inequality implies that $\lim \sup _{\nu \downarrow 0} G(\nu) \leq G(0)$. Thus $\lim _{\nu \downarrow 0} G(\nu)=G(0)$, i.e., $G(\nu)$ is right continuous at $\nu=0$.

By definition, a ground-state of $T(\nu)$ is a non-zero vector $\Psi(\nu)$ in $D(T(\nu))$ such that

$$
T(\nu) \Psi(\nu)=G(\nu) \Psi(\nu)
$$

We assume the following:

(T.2) There exists a constant $\nu_{0} \in(0, c)$ such that, for each $\nu \in\left(0, \nu_{0}\right), T(\nu)$ has a ground-state $\Psi(\nu)$ with $\|\Psi(\nu)\|=1$.

Under the assumptions (T.1) and (T.2), we can define for a linear operator $X$ on $\mathcal{X}$ with $D(X) \supset D(T) \cap D(S)$

$$
\langle X\rangle_{\nu}:=(\Psi(\nu), X \Psi(\nu)), \quad \nu \in\left(0, \nu_{0}\right)
$$

the ground-state expectation value of $X$. 
Lemma A.2 For all $\nu \in\left(0, \nu_{0}\right), \nu^{\prime} \in[0, c)$ with $\nu^{\prime} \neq \nu$,

$$
\langle S\rangle_{\nu}=\frac{\left\langle T\left(\nu^{\prime}\right)\right\rangle_{\nu}-G(\nu)}{\nu^{\prime}-\nu}
$$

Proof. We have

$$
\left(\nu^{\prime}-\nu\right)\langle S\rangle_{\nu}=\left(\Psi(\nu),\left[T\left(\nu^{\prime}\right)-T(\nu)\right] \Psi(\nu)\right)=\left\langle T\left(\nu^{\prime}\right)\right\rangle_{\nu}-G(\nu) .
$$

Hence (A.9) follows.

Proposition A.3 Suppose that $S \geq 0$. Then:

(i) If $D(T) \cap D(S)$ is a core of $T$, then $\lim _{\nu \downarrow 0} \nu\langle S\rangle_{\nu}=0$ and $\lim _{\nu \downarrow 0}\langle T\rangle_{\nu}=G(0)$.

(ii) For all $\nu \in\left(0, \nu_{0}\right)$,

$$
\langle S\rangle_{\nu} \geq G^{\prime}(\nu+0)
$$

(iii) If the right differential

$$
G^{\prime}(0+):=\lim _{\nu \downarrow 0} \frac{G(\nu)-G(0)}{\nu}
$$

of $G(\nu)$ at $\nu=0$ exists, then

$$
\limsup _{\nu \downarrow 0}\langle S\rangle_{\nu} \leq G^{\prime}(0+)
$$

(iv) $\lim \sup _{\nu \downarrow 0}\langle S\rangle_{\nu}<\infty$ if and only if $G(\nu)-\langle T\rangle_{\nu}=O(\nu)(\nu \downarrow 0)$, where $O(\cdot)$ is Landau's symbol.

Proof. (i) By (A.9) with $\nu^{\prime}=0$ and the variational principle, we have

$$
0 \leq \nu\langle S\rangle_{\nu} \leq G(\nu)-G(0)
$$

Hence, by Proposition A.1(iv), we obtain the desired result.

By (A.9) with $\nu^{\prime}=0$, we have

$$
\langle T\rangle_{\nu}=G(\nu)-\nu\langle S\rangle_{\nu}
$$

By part (i) and Proposition A.1(iv), we obtain the desired result.

(ii) Let $c>\nu^{\prime}>\nu>0$. Then, by (A.9) and the variational principle, we have

$$
\langle S\rangle_{\nu} \geq \frac{G\left(\nu^{\prime}\right)-G(\nu)}{\nu^{\prime}-\nu} .
$$

Taking $\nu^{\prime} \downarrow \nu$, we obtain (A.10). 
(iii) By (A.13), we have

$$
\langle S\rangle_{\nu} \leq \frac{G(\nu)-G(0)}{\nu}
$$

from which the desired result follows.

(iv) Let $\lim \sup _{\nu \downarrow 0}\langle S\rangle_{\nu}<\infty$. Then $D_{\delta}:=\sup _{\nu \in(0, \delta)}\langle S\rangle_{\nu}<\infty$ for each sufficiently small constant $\delta<c$. Hence, by Lemma A.2, we have $\left|G(\nu)-\langle T\rangle_{\nu}\right| \leq D_{\delta} \nu, \nu \in(0, \delta)$. Hence $G(\nu)-\langle T\rangle_{\nu}=O(\nu)(\nu \downarrow 0)$.

Conversely, let $G(\nu)-\langle T\rangle_{\nu}=O(\nu)(\nu \downarrow 0)$. Then, for all sufficiently small $\nu>0$, $\left|G(\nu)-\langle T\rangle_{\nu}\right| \leq D \nu$, where $D>0$ is a constant. This implies that $\langle S\rangle_{\nu} \leq D$. Hence $\lim \sup _{\nu \downarrow 0}\langle S\rangle_{\nu} \leq D$.

\section{B Abstract Results on Discrete Spectrum of a Self- Adjoint Operator}

Let $\mathcal{H}$ be a Hilbert space and $H_{1}$ be a self-adjoint operator on $\mathcal{H}$, bounded from below. Let $H_{2}$ be a symmetric operator on $\mathcal{H}$ and

$$
H:=H_{1}+H_{2} \text {. }
$$

We assume the following:

(C.1) $D\left(H_{1}\right) \subset D\left(H_{2}\right), H$ is self-adjoint on $D\left(H_{1}\right)$ and bounded from below.

Under condition (C.1), we define for $r>0$

$$
S_{r}:=\left\{z \in \mathbf{C}|| z-\mathcal{E}_{0}(H) \mid=r\right\} .
$$

We need additional conditions:

(C.2) For all $z \in \varrho\left(H_{1}\right):=\mathbf{C} \backslash \sigma\left(H_{1}\right)$ (the resolvent set of $\left.H_{1}\right), H_{2}\left(H_{1}-z\right)^{-1}$ is a bounded operator on $\mathcal{H}$.

(C.3) For a constant $M>0, H_{1}$ has purely discrete spectrum in $\left[\mathcal{E}_{0}\left(H_{1}\right), \mathcal{E}_{0}\left(H_{1}\right)+M\right)$.

(C.4) There exists a constant $\delta \in(0, M)$ such that

$$
\mathcal{E}_{0}(H) \pm \delta \notin \sigma\left(H_{1}\right), \quad \mathcal{E}_{0}(H)-\mathcal{E}_{0}\left(H_{1}\right)<M-\delta,
$$

and

$$
C_{\delta}:=\sup _{z \in S_{\delta}}\left\|H_{2}\left(H_{1}-z\right)^{-1}\right\|<\frac{1}{1+q_{\delta}}
$$

where

$$
q_{\delta}:=\sup _{z \in S_{\delta}} \sup _{\lambda \in \sigma\left(H_{1}\right)} \frac{1}{|\lambda-z|} .
$$


Theorem B.1 Assume (C.1)-(C.4). Then H has purely discrete spectrum in the interval $\left[\mathcal{E}_{0}(H), \mathcal{E}_{0}(H)+\delta\right)$. In particular, $H$ has a ground state.

Proof. For all $z \in \varrho\left(H_{1}\right)$,

$$
H-z=(I+K(z))\left(H_{1}-z\right)
$$

with $K(z):=H_{2}\left(H_{1}-z\right)^{-1}$. Let $z \in S_{\delta}$. Then, by (C.4), $z \in \varrho\left(H_{1}\right)$ and $I+K(z)$ is bijective. Hence $z \in \varrho(H)$ with

$$
(H-z)^{-1}-\left(H_{1}-z\right)^{-1}=\sum_{n=1}^{\infty}(-1)^{n}\left(H_{1}-z\right)^{-1} K(z)^{n},
$$

where the convergence is taken in operator norm topology. For a self-adjoint operator $T$, we denote by $P_{T}$ its spectral measure. Let $I_{\delta}:=\left[\mathcal{E}_{0}(H), \mathcal{E}_{0}(H)+\delta\right)$. Then we have

$$
P_{H}\left(I_{\delta}\right)=(-2 \pi i)^{-1} \int_{S_{\delta}}(H-z)^{-1} d z, \quad P_{H_{1}}\left(I_{\delta}\right)=(-2 \pi i)^{-1} \int_{S_{\delta}}\left(H_{1}-z\right)^{-1} d z .
$$

By (C.3) and (C.4), $\operatorname{dim} \operatorname{Ran} P_{H_{1}}\left(I_{\delta}\right)<\infty$. By (B.4), we have

$$
\left\|P_{H}\left(I_{\delta}\right)-P_{H_{1}}\left(I_{\delta}\right)\right\| \leq q_{\delta} \sum_{n=1}^{\infty} C_{\delta}^{n}=\frac{q_{\delta} C_{\delta}}{1-C_{\delta}}<1 .
$$

Hence, by [43, p.14, Lemma], $\operatorname{dim} \operatorname{Ran} P_{H}\left(I_{\delta}\right)=\operatorname{dim} \operatorname{Ran} P_{H_{1}}\left(I_{\delta}\right)<\infty$. Thus the desired result follows.

Let

$$
\mathcal{E}_{1}\left(H_{1}\right):=\inf \sigma\left(H_{1}\right) \cap\left(\mathcal{E}_{0}\left(H_{1}\right), \infty\right)
$$

Then

$$
\mathcal{E}_{1}\left(H_{1}\right)>\mathcal{E}_{0}\left(H_{1}\right)
$$

Let

$$
\delta:=\frac{\mathcal{E}_{0}\left(H_{1}\right)+\mathcal{E}_{1}\left(H_{1}\right)}{2}-\mathcal{E}_{0}(H)
$$

Corollary B.2 Assume (C.1) and (C.3). Suppose that there exist constants $a, b \geq 0$ such that, for all $\Psi \in D\left(H_{1}\right)$,

$$
\left\|H_{2} \Psi\right\| \leq a\left\|H_{1} \Psi\right\|+b\|\Psi\| .
$$

Moreover, suppose that

$$
\mathcal{E}_{0}\left(H_{1}\right) \geq \mathcal{E}_{0}(H)
$$

and

$$
a+\frac{2\left[b+a\left(\delta+\left|\mathcal{E}_{0}(H)\right|\right)\right]}{\mathcal{E}_{1}\left(H_{1}\right)-\mathcal{E}_{0}\left(H_{1}\right)}<\frac{\mathcal{E}_{1}\left(H_{1}\right)-\mathcal{E}_{0}\left(H_{1}\right)}{\mathcal{E}_{1}\left(H_{1}\right)-\mathcal{E}_{0}\left(H_{1}\right)+2} .
$$

Then $H$ has purely discrete spectrum in the interval $\left[\mathcal{E}_{0}(H), \mathcal{E}_{0}(H)+\delta\right)$. In particular, $H$ has a ground state. 
Proof. Condition (B.8) implies that, for all $z \in \varrho\left(H_{1}\right), H_{2}\left(H_{1}-z\right)^{-1}$ is bounded with

$$
\begin{aligned}
\left\|H_{2}\left(H_{1}-z\right)^{-1}\right\| & \leq a\left\|H_{1}\left(H_{1}-z\right)^{-1}\right\|+b\left\|\left(H_{1}-z\right)^{-1}\right\| \\
& \leq a \sup _{\lambda \in \sigma\left(H_{1}\right)} \frac{|\lambda|}{|\lambda-z|}+b \sup _{\lambda \in \sigma\left(H_{1}\right)} \frac{1}{|\lambda-z|} \\
& \leq a \sup _{\lambda \in \sigma\left(H_{1}\right)}\left(1+\frac{|z|}{|\lambda-z|}\right)+b \sup _{\lambda \in \sigma\left(H_{1}\right)} \frac{1}{|\lambda-z|} .
\end{aligned}
$$

Hence (C.2) is fulfilled. By (B.9), we have $\delta>0$. Then it is easy to see that

$$
\begin{gathered}
\sup _{z \in S_{\delta}, \lambda \in \sigma\left(H_{1}\right)} \frac{|z|}{|\lambda-z|} \leq \frac{\delta+\left|\mathcal{E}_{0}(H)\right|}{\left|\mathcal{E}_{0}\left(H_{1}\right)-\mathcal{E}_{0}(H)-\delta\right|}=\frac{2\left(\delta+\left|\mathcal{E}_{0}(H)\right|\right)}{\mathcal{E}_{1}\left(H_{1}\right)-\mathcal{E}_{0}\left(H_{1}\right)}, \\
q_{\delta}=\sup _{z \in S_{\delta}, \lambda \in \sigma\left(H_{1}\right)} \frac{1}{|\lambda-z|}=\frac{1}{\left|\mathcal{E}_{0}\left(H_{1}\right)-\mathcal{E}_{0}(H)-\delta\right|}=\frac{2}{\mathcal{E}_{1}\left(H_{1}\right)-\mathcal{E}_{0}\left(H_{1}\right)} .
\end{gathered}
$$

Hence it follows that

$$
\sup _{z \in S_{\delta}}\left\|H_{2}\left(H_{1}-z\right)^{-1}\right\| \leq a+\frac{2\left[b+a\left(\delta+\left|\mathcal{E}_{0}(H)\right|\right)\right]}{\mathcal{E}_{1}\left(H_{1}\right)-\mathcal{E}_{0}\left(H_{1}\right)}<\frac{1}{1+q_{\delta}} .
$$

Hence (C.4) is satisfied. Thus, applying Theorem B.1, we obtain the desired result.

\section{References}

[1] Amann, A.: Chirality: a superselection rule generated by the molecular environment?, J. Math. Chemistry 6 (1991), 1-15.

[2] Amrein, W. O., Jauch, J. M. and Sinha, K. B.: Scattering Theory in Quantum Mechanics, Benjamin, Reading, Massachusetts, 1977.

[3] Arai, A.: Self-adjointness and spectrum of Hamiltonians in nonrelativistic quantum electrodynamics, J. Math. Phys. 22 (1981), 534-537.

[4] Arai, A.: On a model of a harmonic oscillator coupled to a quantized, massless, scalar field I, J. Math. Phys. 22 (1981), 2539-2548.

[5] Arai, A.: Rigorous theory of spectra and radiation for a model in quantum electrodynamics, J. Math. Phys. 24 (1983), 1896-1910.

[6] Arai, A.: A note on scattering theory in non-relativistic quantum electrodynamics, J. Phys. A: Math. Gen. 16 (1983), 49-70.

[7] Arai, A.: Spectral analysis of a quantum harmonic oscillator coupled to infinitely many scalar bosons, J. Math. Anal. Appl. 140 (1989), 270-288. 
[8] Arai, A.: Long-time behavior of two-point functions of a quantum harmonic oscillator interacting with bosons, J. Math. Phys. 30 (1989), 127-1288.

[9] Arai, A.: An aymptotic analysis and its application to the nonrelativistic limit of the Pauli-Fierz and a spin-boson model, J. Math. Phys. 31 (1990), 2653-2663.

[10] Arai, A.: Noninvertible Bogoliubov transformations and instability of embedded eigenvalues, J. Math. Phys. 32 (1991), 1834-1846.

[11] Arai, A.: Long-time behavior of an electron interacting with a quantized radiation field, J. Math. Phys. 32 (1991), 2224-2242.

[12] Arai, A.: A class of representations of the *-algebra of the canonical commutation relations over a Hilbert space and instability of embedded eigenvalues in quantum field models, J. Nonlinear Math. Phys. 4 (1997), 338-349.

[13] Arai, A.: Introduction to Mathematical Methods of Quantum Field Theory (in Japanese), Lecture Notes Series in Mathematics Vol. 5, Osaka University, Osaka Mathematical Publications, Osaka, 1997.

[14] Arai, A.: Essential spectrum of a self-adjoint operator on an abstract Hilbert space of Fock type and applications to quantum field Hamiltonians, Hokkaido University Preprint Series in Mathematics \#445, February, 1999.

[15] Arai, A. and Hirokawa, M.: On the existence and uniqueness of ground states of a generalized spin-boson model, J. Funct. Anal. 151 (1997), 455-503.

[16] Arai, A., Hirokawa, M. and Hiroshima, F.: On the absence of eigenvectors of Hamiltonians in a class of massless quantum field models without infrared cutoff, Hokkaido University Preprint Series in Mathematics \#440, December, 1998.

[17] Bach, V., Fröhlich, J. and Sigal, I. M.: Quantum electrodynamics of confined nonrelativistic particles, Adv. in Math. 137 (1998), 299-395.

[18] Bach, V., Fröhlich, J. and Sigal, I. M.: Spectral Analysis for Systems of Atoms and Molecules coupled to the Quantized Radiation Field, preprint, 1998.

[19] Billionnet, C.: About poles of the resolvent, in a model for a harmonic oscillator coupled with massless bosons, Ann. Inst. Henri Poincaré 68 (1998), 1-16.

[20] Blanchard, P.: Discussion mathématique du modéle de Pauli et Fierz relatif á la catastrophe infrarouge, Commun. Math. Phys. 15 (1969), 156-172.

[21] Bloch, F. and Nordsieck, A.: Notes on the radiation field of the electron, Phys. Rev. 52 (1937), 54-59. 
[22] Davies, E. B.: Symmetry breaking for molecular open system, Ann. Inst. Henri Poincaré A 35 (1981), 149-171.

[23] Davies, E. B.: Dynamics of a multilevel Wigner-Weisskopf atom, J. Math. Phys. 15 (1974), 2036-2041.

[24] Dereziński, J. and Jakšić, V.: Spectral theory of Pauli-Fierz Hamiltonians I, preprint, December, 1998.

[25] Emch, G. G.: Algebraic Methods in Statistical Mechanics and Quantum Field Theory, John Wiley \& Sons, New York, 1972.

[26] Friedrichs, K. O.: On the perturbation of continuous spectra, Comm. Pure Appl. Math. I (1948), 361-406.

[27] Fröhlich, J.: On the infrared problem in a model of scalar electrons and massless, scalar bosons, Ann. Inst. Henri Poincaré 19 (1973), 1-103.

[28] Fröhlich, J.: Existence of dressed one electron states in a class of persistent models, Fortschr. der Phys. 22 (1974), 159-198.

[29] Gross, E. P.: Ground state of a spin-phonon system I. Variational estimates, J. Stat. Phys. 54 (1989), 405-427.

[30] Hirokawa, M.: An expression of the ground state energy of the spin-boson model, $J$. Funct. Anal. 162 (1999), 178-218.

[31] Hiroshima, F.: Ground states and spectrum of non-relativistic particles in quantum electrodynamics, preprint, 1998.

[32] Hiroshima, F.: Ground states of a model in quantum electrodynamics, preprint, 1998

[33] Hiroshima, F.: Uniqueness of the ground state of a model in quantum electrodynamics: a functional integral approach, preprint, 1998.

[34] Hübner, M. and Spohn, H.: Radiative decay: Nonperturbative approaches, Rev. Math. Phys. 7 (1995), 363-387.

[35] Hübner, M. and Spohn, H.: Spectral properties of the spin-boson Hamiltonian, Ann. Inst. Henri. Poincaré 62 (1995), 289-323.

[36] Itzykson, C. and Zuber, J.-B.: Quantum Field Theory, McGraw-Hill, New York, 1980.

[37] Ikromov, I. A. and Sharipov, F.: On the discrete spectrum of the nonanalytic matrixvalued Friedrichs model, Funct. Anal. Appl. 32 (1998), 49-51. 
[38] King, C.: Exponential decay near resonance, without analyticity, Lett. Math. Phys. 23(1991), 215-222.

[39] Pauli, W. and Fierz, M.: Zur Theorie der Emission langwelliger Lichtquanten, Nuovo Cimento 15 (1938), 167-188.

[40] Pfeifer, P.: Chiral molecules - a superselection rule induced by the radiation field, Dissertation, ETH-Zürich, No.6551, ok Gotthard S + D AG, Zürich, 1980.

[41] Reed, M. and Simon, B.: Methods of Modern Mathematical Physics Vol. I, Academic Press, New York, 1972.

[42] Reed, M. and Simon, B.: Methods of Modern Mathematical Physics Vol. II, Academic Press, New York, 1975.

[43] Reed, M. and Simon, B.: Methods of Modern Mathematical Physics Vol. IV, Academic Press, New York, 1978.

[44] Spohn, H: Ground state(s) of the spin-boson Hamiltonian, Commun. Math. Phys. 123 (1989), 277-304.

[45] Spohn, H.: Asymptotic completeness for Rayleigh scattering, J. Math. Phys. 38 (1997), 2281-2296.

[46] Tsuzuki, T.: Ground state of a spin-boson system II. Wavefunction and variational study, Prog. Theo. Phys. 87 (1992), 569-581. 